\title{
Motor Circuit-Specific Burst Patterns Drive Different Muscle and Behavior Patterns
}

\author{
Florian Diehl, ${ }^{1,3 \star}$ Rachel S. White, ${ }^{1 \star}$ Wolfgang Stein, ${ }^{2,3}$ and Michael P. Nusbaum ${ }^{1}$ \\ ${ }^{1}$ Department of Neuroscience, Perelman School of Medicine, University of Pennsylvania, Philadelphia, Pennsylvania 19104, ${ }^{2}$ School of Biological Sciences, \\ Illinois State University, Normal, Illinois 61790, and ${ }^{3}$ Institute of Neurobiology, Ulm University, 89069 Ulm, Germany
}

In the isolated CNS, different modulatory inputs can enable one motor network to generate multiple output patterns. Thus far, however, few studies have established whether different modulatory inputs also enable a defined network to drive distinct muscle and movement patterns in vivo, much as they enable these distinctions in behavioral studies. This possibility is not a foregone conclusion, because additional influences present in vivo (e.g., sensory feedback, hormonal modulation) could alter the motor patterns. Additionally, rhythmic neuronal activity can be transformed into sustained muscle contractions, particularly in systems with slow muscle dynamics, as in the crab (Cancer borealis) stomatogastric system used here. We assessed whether two different versions of the biphasic (protraction, retraction) gastric mill (chewing) rhythm, triggered in the isolated stomatogastric system by the modulatory ventral cardiac neurons (VCNs) and postoesophageal commissure (POC) neurons, drive different muscle and movement patterns. One distinction between these rhythms is that the lateral gastric (LG) protractor motor neuron generates tonic bursts during the VCN rhythm, whereas its POC-rhythm bursts are divided into fast, rhythmic burstlets. Intracellular muscle fiber recordings and tension measurements show that the LGinnervated muscles retain the distinct VCN-LG and POC-LG neuron burst structures. Moreover, endoscope video recordings in vivo, during VCN-triggered and POC-triggered chewing, show that the lateral teeth protraction movements exhibit the same, distinct protraction patterns generated by LG in the isolated nervous system. Thus, the multifunctional nature of an identified motor network in the isolated CNS can be preserved in vivo, where it drives different muscle activity and movement patterns.

\section{Introduction}

In the isolated CNS, individual neural networks, such as central pattern generators (CPGs), can generate different output patterns in response to different modulatory inputs (Marder and Calabrese, 1996; Marder and Bucher, 2001; Dickinson, 2006; Doi and Ramirez, 2008; Harris-Warrick, 2011; Marder, 2012). Changes in motor patterns commonly modify movement by altering the amplitude and relative timing of muscle activity (Kiehn and Kjaerulff, 1996; Thuma et al., 2003; Wenning et al., 2004; Zhurov et al., 2005; Rosenbaum et al., 2010). Modulated neural activity patterns can also differ in their burst structure, but less is known regarding whether this distinction also occurs in vivo and remains distinct in the associated muscles and movements (Hooper et al., 2006; Zhurov and Brezina, 2006). This outcome is

Received March 11, 2013; revised May 23, 2013; accepted June 12, 2013.

Author contributions: F.D., R.S.W., W.S., and M.P.N. designed research; F.D. and R.S.W. performed research; F.D.,

R.S.W., W.S., and M.P.N. analyzed data; F.D., R.S.W., W.S., and M.P.N. wrote the paper.

*F.D. and R.S.W. contributed equally to this work.

This work was supported by National Institute of Neurological Disease and Stroke Grant R37-NS29436 (M.P.N.), German Research Foundation Grant DFG STE 937/7-1 and 8-1 (W.S.), and German Academic Exchange Fellowship DAAD-D/11/43651 (F.D.). We thank Drs. Dawn M. Blitz and Farzan Nadim for informative discussions, and Dr. Joshua I. Gold for assistance with statistical analysis.

Correspondence should be addressed to Michael P. Nusbaum, PhD, Dept. of Neuroscience, Perelman School of Medicine, University of Pennsylvania, 215 Stemmler Hall, Philadelphia, PA 19104-6074. E-mail: nusbaum@ mail.med.upenn.edu.

R.S. White's present address: Department of Psychiatry, Perelman School of Medicine, University of Pennsylvania, Philadelphia, PA 19104-3403.E-mail: whiters@mail.med.upenn.edu.

DOI:10.1523/JNEUROSCI.1060-13.2013

Copyright $\odot 2013$ the authors $\quad 0270-6474 / 13 / 3312013-17 \$ 15.00 / 0$ not a foregone conclusion, because sensory feedback and hormonal modulation can alter motor patterns and muscle properties, and rhythmic activity can be modified by muscle dynamics (Carrier, 1989; Hooper and Weaver, 2000; Hooper et al., 2006; Zhurov and Brezina, 2006) and the musculoskeletal system (Prochazka and Yakovenko, 2007; O'Connor et al., 2012).

We examined this issue using different versions of the biphasic (protraction, retraction) gastric mill (chewing) rhythm in Cancer borealis, an omnivorous crab species that ingests various hardbodied and soft-bodied species and thus likely requires different chewing patterns (Stehlik, 1993; Donahue et al., 2009). We focused on the well characterized gastric mill rhythms triggered by two modulatory inputs, the ventral cardiac neurons (VCNs) and postoesophageal commissure (POC) neurons (White and Nusbaum, 2011). In the isolated stomatogastric nervous system (STNS), stimulating either pathway triggers long-lasting, distinct activity patterns in the same two commissural ganglia (CoGs) projection neurons, which then drive the gastric mill CPG in the stomatogastric ganglion (STG; Beenhakker and Nusbaum, 2004; Beenhakker et al., 2004; Blitz et al., 2008). One prominent difference between these rhythms is that the lateral gastric (LG) protractor neuron burst structure is tonic after VCN stimulation, but exhibits a series of brief burstlets after POC stimulation (Blitz and Nusbaum, 2008, 2012; White and Nusbaum, 2011).

Here we determined whether the different LG burst structures during the VCN-triggered and POC-triggered gastric mill rhythms are reliably maintained by the lateral tooth protractor muscles and the resulting protraction movements during chew- 
ing. We first established, in semi-intact preparations, that three different LGinnervated muscles (gm5b, gm6ab, gm8a) exhibit VCN-specific and POC-specific excitatory junction potential (EJP) patterns and, in at least one of these muscles (gm6ab), tension patterns. The gm6ab muscle also exhibited larger and more sustained tension increases during the VCN rhythm, likely due to the VCNspecific LG burst structure. During chewing movements in vivo, driven either by stimulating the gastric mill motor neurons with in vitro VCN and POC-motor patterns or by triggering free running VCN- and POC-gastric mill rhythms, the lateral teeth protraction movements were VCN and POC specific. These data support the hypothesis that distinct versions of a centrally generated motor pattern from a single motor network in the isolated CNS can drive different versions of the resulting behavior, even in a system with slow muscle dynamics.

\section{Materials and Methods}

Animals. Male Jonah crabs (Cancer borealis) were obtained from commercial suppliers (Yankee Lobster; Marine Biological Laboratory), and maintained in aerated, filtered artificial seawater at $10-12^{\circ} \mathrm{C}$. Animals were cold anesthetized by packing them in ice for at least $30 \mathrm{~min}$ before dissection. For muscle fiber and muscle tension recordings, the foregut was then removed from the animal and the remaining dissection of the STNS plus specific foregut muscles was performed in physiological saline at $\sim 8^{\circ} \mathrm{C}$ (see below for the in vivo preparation protocol).

Solutions. C. borealis physiological saline contained the following (in $\mathrm{mm}$ ): $440 \mathrm{NaCl}, 26$ $\mathrm{MgCl}_{2}, 13 \mathrm{CaCl}_{2}, 11 \mathrm{KCl}, 10$ Trisma base, 5 maleic acid, 5 dextrose, $\mathrm{pH}$ 7.4-7.6. All preparations were superfused continuously with $C$. borealis saline $\left(8-12^{\circ} \mathrm{C}\right)$.

Electrophysiology. Electrophysiology experiments were performed using standard techniques for this system (Weimann et al., 1991; Beenhakker et al., 2004). For muscle fiber recordings, the STNS plus the LG-innervated muscles (Fig. $1 A, B$ ) were pinned down in a silicone elastomer-lined (Sylgard 184, K.R. Anderson) Petri dish. All STG motor nerve branches except the bilaterally symmetric lateral gastric nerves (lgns; Fig. 1) were bisected, preventing all motor neurons except LG neurons from regulating their muscle targets. There is a single LG motor neuron per STG in C. borealis (Kilman and Marder, 1996).

Extracellular recordings were made by pressing one of a pair of stainless steel wire electrodes into the Sylgard adjacent to an identified nerve and isolating that electrode and region of nerve from the main bath compartment with petroleum jelly (Vaseline, Medical Accessories and Supply Headquarters). The second electrode of each pair was pressed into the Sylgard
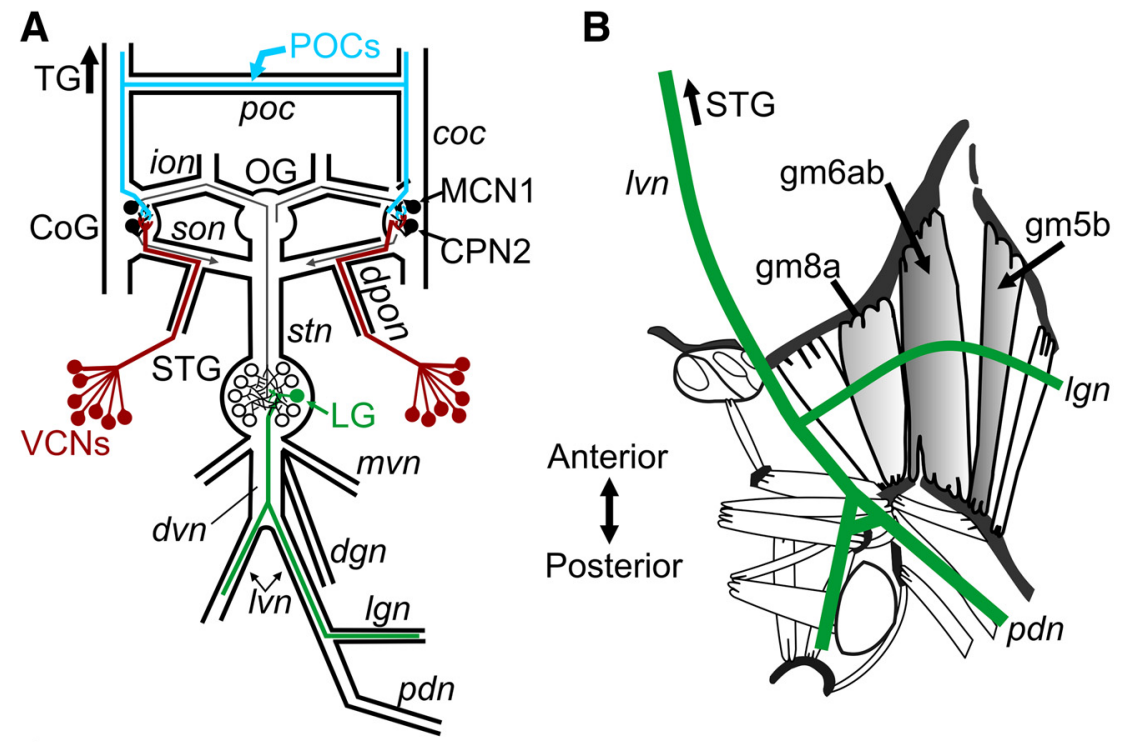

C
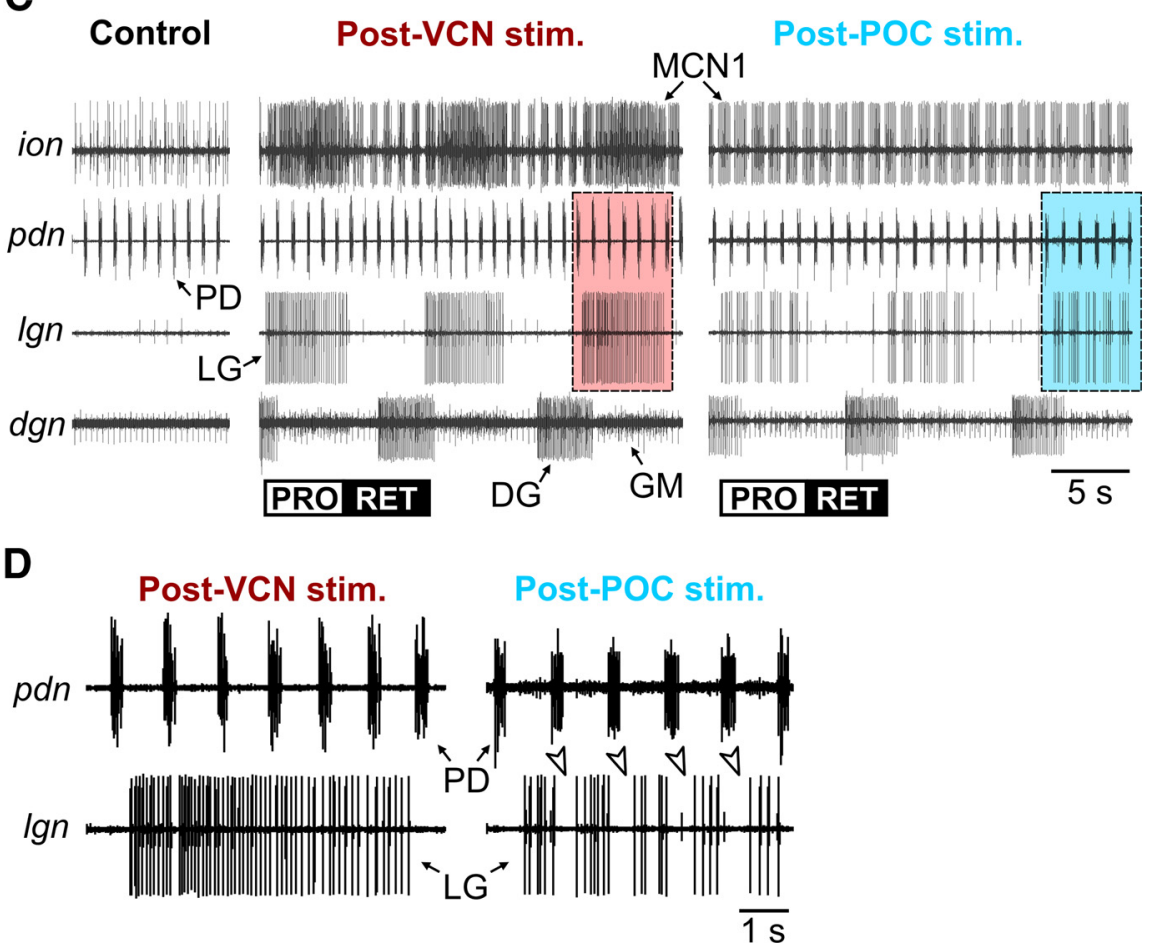

Figure 1. The gastric mill protraction neuron $L G$ generates different activity patterns during the VCN-triggered and POCtriggered gastric mill rhythm. $\boldsymbol{A}$, Schematic of the isolated stomatogastric nervous system, including its four ganglia plus the connecting nerves and a subset of peripheral nerves. The VCNs project into the CoGs from the cardiac sac stomach compartment via the dpon and superior oesophageal nerve (son). The POC neurons project into the CoGs via the poc nerve and the cocs (coc). The LG neuron axon projects through the $d v n$, Ivns, and Igns to innervate its muscle targets (Fig. 1B). OG, oesophageal ganglion; TG, thoracic ganglion; ion, inferior oesophageal nerve; $m v n$, medial ventricular nerve; $p d n$, pyloric dilator nerve; stn, stomatogastric nerve. $\boldsymbol{B}$, Schematic dorsal view of the posterior region (right half) of a dissected C. borealis foregut (modified from Weimann et al., 1991). LG innervates several protractor muscles (gray shading), including gm8a, gm6ab, and gm5b, via the /vn and Ign. C, Gastric mill motor patterns triggered by brief stimulation of the POC and VCN pathways and recorded extracellularly from nerves shown schematically in $\boldsymbol{A}$. No gastric mill rhythm was in progress before either pathway was stimulated, but the pyloric rhythm was ongoing ( $p d n$, control). Note that the MCN1 and LG neuron burst patterns were pyloric rhythm-timed (see $p d n$ ) during the POC-triggered gastric mill rhythm but were tonic during the protraction phase of the VCN-triggered gastric mill rhythm. The POC-triggered and VCN-triggered gastric mill rhythms were recorded in separate preparations. The control recording shown was from the POC preparation. Boxed regions are expanded in D. PRO, Protraction; RET, retraction. Modified from White and Nusbaum (2011). D, Expanded region from Cof the $p d n$ and Ign recordings, showing the lack of pyloric (PD)-timed activity within the LG burst during the VCN-triggered gastric mill rhythm (Post-VCN stim.) and the presence of pyloric-timed interruptions within the LG burst (arrowheads) during the POC rhythm (Post-POC stim.). 
in the electrically grounded main bath compartment. Extracellular nerve stimulation was accomplished by placing the pair of wires used to record nerve activity into a stimulus isolation unit (Model SIU5, Astro-Med/ Grass Instruments) that was connected to a stimulator (Model S88, Astro-Med/Grass Instruments).

Intracellular muscle fiber recordings were made with microelectrodes $\left(10-15 \mathrm{M} \Omega\right.$ ) filled with $0.6 \mathrm{M} \mathrm{K}_{2} \mathrm{SO}_{4}$ plus $10 \mathrm{~mm} \mathrm{KCl}$. To facilitate intracellular recordings, the exposed muscle fibers were viewed with light transmitted through a dark-field condenser (Nikon). Intracellular signals were amplified using Axoclamp 2B amplifiers (Molecular Devices), then further amplified and filtered with Brownlee 410 amplifiers (Brownlee Precision) and digitized using a Micro 1401 or Power 1401 data acquisition interface and Spike2 software (version 7.0-7.11; Cambridge Electronic Design). Muscles solely innervated by LG (Fig. 1B, gm5b, gm6ab, gm8a) were identified on the basis of their location in the gastric mill stomach compartment, position relative to identified nerves and presence of LG neuron-mediated EJPs or tension increases (Hooper et al., 1986; Weimann et al., 1991; Stein et al., 2006). To minimize movement artifacts and prematurely lost recordings due to muscle contractions, intracellular muscle fiber recordings were obtained close to one of the muscle attachment points after removing the connective tissue from that region of the muscle.

POC neuron stimulation sufficient to trigger the gastric mill rhythm was accomplished by electrically stimulating one or both halves of the bisected poc (Fig. 1A), using tonic stimulation (frequency, $15-30 \mathrm{~Hz}$; duration, 15-30 s; Blitz et al., 2008). Threshold for extracellular activation of the POC neurons was determined by monitoring the activity of the projection neuron modulatory commissural neuron 1 (MCN1) in the ipsilateral inferior oesophageal nerve (Fig. 1A, ion). The VCNs were activated by stimulating the dorsal posterior oesophageal nerve (dpon; Fig. 1A). The dpon stimulations were done using a rhythmic pattern (burst duration, $6 \mathrm{~s}$; interburst frequency, $0.06 \mathrm{~Hz}$; intraburst frequency, $15 \mathrm{~Hz}$; Beenhakker et al., 2004). It is noteworthy, however, that the rhythmic dpon stimulation pattern triggers the same gastric mill rhythm as tonic or pyloric rhythm-like stimulation patterns (Beenhakker et al., 2004). To avoid cross-pathway influences resulting from long-lasting modulator actions, we elicited POC-induced and $\mathrm{VCN}$-induced gastric mill rhythms in different preparations.

Muscle-tension recordings of the LG-innervated gm6ab muscle were obtained using an isometric force transducer (Harvard Apparatus) on the isolated gm6ab muscle and the cartilaginous ossicles to which it attaches (Pearson, 1908; Maynard and Dando, 1974). To obtain the isolated but still innervated gm6ab muscle, the gastropyloric stomach region was bisected rostrocaudally, with one side remaining innervated by the STG motor nerves. The innervated side was then pinned down onto the Sylgard. The gm6ab muscle on the innervated side was then isolated by bisecting all other gastric mill and pyloric muscles and secured with pins at its anterior insertion site, the zygocardiac ossicle (Pearson, 1908; Maynard and Dando, 1974), without damaging the muscle or its innervation [lateral ventricular nerve (lvn, Fig. 1B) to $\lg n$ (Fig. 1B)]. This muscle was then stretched vertically to its original length and attached via the inferior lateral cardiac ossicle to the force transducer. The $l v n$ was stimulated using LG activity patterns that were recorded during POCinduced and VCN-induced gastric mill rhythms in the isolated STNS and converted to stimulation patterns ("realistic patterns") using a customwritten script (Convert_event_to_sequencer.s2s) for the Spike2 data acquisition and analysis system (Cambridge Electronic Design). This script is freely available at http://www.neurobiologie.de/spike2. These realistic stimulation patterns drove the S88 Stimulator to provide unitary, suprathreshold stimuli (duration, $1 \mathrm{~ms} /$ stimulus) to the $l v n$ that elicited LG action potentials. Muscle-tension measurements were recorded and stored on computer using the Spike2 software. For the realistic stimulations, LG activity patterns were obtained from three different preparations for each type of gastric mill rhythm (see Results).

Extracellular recordings and stimulations of LG in the animal were performed using a hook electrode on the dorsal ventricular nerve $(d v n)$ or $l v n$, with the reference electrode inserted into the abdominal cavity (Hedrich and Stein, 2008; Hedrich et al., 2011). We use the phrase "in situ" to represent experiments performed in the otherwise intact, re- strained crab where tooth movements were driven by $d v n$ or $l v n$ stimulation, coordinated with dorsal gastric nerve $(d g n)$ stimulation as described below, using the aforementioned realistic VCN and POC patterns. In the in situ experiments, the aforementioned nerves were bisected, and nerve stimulations were performed using the section of the cut nerve that remained connected to the relevant muscles.

To optimize selective LG stimulation in situ via the $d v n$ or $l v n$, the stimulation voltage was adjusted in each experiment to be just above threshold for eliciting lateral tooth protraction movements. The LG neuron has the largest diameter axon in these nerves, as indicated by its exhibiting the largest amplitude action potential in extracellular $d v n$ and $l v n$ recordings, and thus has the lowest threshold for extracellular stimulation. The other lateral teeth protractor motor neuron [medial gastric (MG) neuron; Weimann et al., 1991], also projects its axon through the $d v n$ and $l v n$, but was unlikely to have been activated by these stimulations because it has a considerably smaller axon diameter and smaller amplitude extracellular action potential. To enable coordinated activation of the medial and lateral teeth in VCN-like and POC-like patterns during the in situ experiments, we costimulated LG and the medial tooth protractor [gastric mill (GM) neuron] and retractor [dorsal gastric (DG) neuron] motor neurons. To stimulate the axons of the medial tooth motor neurons, we bisected the $d g n$ and stimulated its anterior branch to drive GM neuron activity, while stimulating its posterior branch to drive DG activity. The GM and DG neurons are the only motor neurons in the dgn.

In vivo/in situ experimental preparation. Crabs were first anesthetized by immersion in ice for at least $30 \mathrm{~min}$, after which they were restrained with rubber bands and fixed into a custom-made, ice-filled Plexiglas box (Heinzel et al., 1993). The mouthparts were then removed to reveal the mandibles, which were clipped to enable endoscope access to the oesophagus. To keep the gills moist, a small $(0.5 \mathrm{~cm})$ saline-soaked cellulose strip was rolled up and inserted into each pterygostoma.

The phrase "in vivo" is used to represent experiments performed on fully intact and restrained crabs where gastric mill rhythms were elicited via either mechanical stimulation of the cardiac gutter or via electrical stimulation of the circumoesophageal connective (coc) or poc. To expose the $c o c$ and poc for in vivo experiments, the ventral carapace was opened rostrocaudally, from the first to fourth thoracic sternite, and mediolaterally to the coxae of the first pereopods. A small rotary drill (Proxxon) was used to cut through the carapace. During surgery, a syringe was used to continuously provide fresh chilled saline to keep the open region moist. The syringe was kept in place by a lockable ball-joint mount screwed to the crab holder. The ventral thoracic artery was bisected to allow access to the $c o c$ and $p o c$, one of which was then set up for stimulation using a custom-made extracellular hook electrode (Hedrich et al., 2011). The electrode was fastened to lockable ball-joint mounts screwed to the crab holder. The wound was then closed with petroleum jelly.

Cardiac gutter stimulation. The cardiac gutter, located ventromedially in the posterior cardiac sac stomach region (Beenhakker et al., 2004), was lightly stroked with a small diameter probe $(<0.2 \mathrm{~mm})$ inserted into the cardiac sac in parallel to the endoscope. This region was stroked repetitively until a gastric mill rhythm was initiated. If no gastric mill rhythm was initiated within $\sim 30 \mathrm{~s}$, the stimulation was terminated.

Video monitoring of tooth movement. To record tooth movement in the gastric mill stomach compartment, an endoscope (probe diameter, 5.3 $\mathrm{mm}$; view angle. $30^{\circ}$; LED-light, TECAM-3, Richard Wolf $\mathrm{GmbH}$ ) was inserted through the oesophagus and cardiac sac into the anterior gastric mill. The endoscope probe was positioned such that the gastric mill teeth (2 lateral teeth and 1 medial tooth) were fully visible. The camera recorded the video signal in PAL (sampling rate: $25 \mathrm{~Hz}, 640 \times 480$ pixels) and was synchronized with the electrophysiological recording using Spike2. The endoscope camera was mounted to a tripod that was placed near the crab.

The video data were translated into two-dimensional data for further analysis, using auto-tracking software written for Matlab (Mathworks; Hedrick, 2008). This software tracks areas of high contrast when they move in reference to a low-contrast background. Here the black, highcontrast tips of the lateral teeth and the tip of the medial tooth were used for tracking. When available, small markings or other regions of high 
contrast on the teeth were also used. Movements of these high-contrast areas were determined via an extended Kalman filter (Fahrmeir, 1992; Pillow et al., 2011). To compensate for noise resulting from the Kalman filtering process, movement traces were low-pass filtered using a sliding window average with a time base of $120 \mathrm{~ms}$.

The $X$ and $Y$ coordinates for each video frame were saved to file and imported into Spike2. Because endoscope position, view angle, and animal size varied, relative units were used to quantify movements. Specifically, $X$ and $Y$ pixel coordinates were normalized to the widest point of the medial tooth ["medial tooth unit" (MT); Heinzel, 1988a,b; Heinzel et al., 1993].

Data analysis. Electrophysiology data were collected in parallel on chart recorder (Everest model, Astro-Med) and computer, via Spike2, with a sampling rate of $5 \mathrm{kHz}$. Some data analyses, including the instantaneous EJP frequency in the LG-innervated muscles, were conducted on the digitized data with a custom-written Spike2 program called "The Crab Analyzer" (freely available at http://www.neurobiologie.de/spike2). Data were plotted with Excel (version 2003, Microsoft). Final figures were produced using CorelDraw (version 12.0).

Unless otherwise stated, each data point in a dataset was derived by determining the mean for the analyzed parameter from 10 consecutive gastric mill cycles during the steady-state region of the motor pattern, starting $\sim 30-60 \mathrm{~s}$ after gastric mill rhythm onset. One gastric mill cycle is defined as extending from the onset of consecutive LG neuron action potential bursts (Beenhakker and Nusbaum, 2004; Wood et al., 2004). The protractor phase duration was determined by the LG burst duration and, in vivo, the lateral teeth protraction duration. The gastric mill rhythm-timed LG burst duration was defined as the interval between the first and last action potential within an impulse burst during which no interspike interval was longer than $2 \mathrm{~s}(\sim 2 \times$ the pyloric cycle period and briefer than the duration of each gastric mill phase; Beenhakker et al., 2004). The lateral teeth protraction duration was measured from the initial inflection of the tooth movement trajectory, elicited by LG neuron burst onset, to the inflection point at which the trajectory returned to baseline, at the end of the LG burst. The retractor phase duration was determined by measuring the LG interburst interval. Instantaneous EJP frequency, determined for 10 consecutive steady-state bursts during each gastric mill rhythm, was defined as 1 divided by the inter-EJP interval.

We determined the relationship between the EJP burst pattern in the LG-innervated gm6ab muscle and the pyloric rhythm during the POCgastric mill rhythm by measuring the EJP distribution relative to normalized pyloric cycles, as done previously for the LG neuron burst pattern during the POC-gastric mill rhythm (White and Nusbaum, 2011; Blitz and Nusbaum, 2012). The pyloric cycle period $(\sim 1 \mathrm{~s})$ is briefer than the gastric mill-timed LG burst duration $(\sim 5 \mathrm{~s})$, so there are several pyloric cycles per gastric mill protractor phase (Blitz et al., 2008; White and Nusbaum, 2011). For this analysis we monitored the pyloric rhythm via the activity of the projection neuron MCN1, which is pyloric-timed during the POC-gastric mill rhythm (Blitz et al., 2008; Blitz and Nusbaum, 2012). We used MCN1 to monitor the pyloric rhythm because the STG peripheral nerves that are normally used to monitor pyloric motor neuron activity were unavailable, due to the need to preserve the gastric mill and pyloric stomach regions to ensure that the LG neuron innervated its muscle targets. Each normalized pyloric cycle extended from the start of a MCN1 burst ("0") to the start of the next MCN1 burst ("1"). Specifically, for 10 consecutive gastric mill cycles per experiment, we separated the gm6ab recording during each normalized pyloric cycle into 50 equal bins ( 1 bin $=2 \%$ normalized pyloric cycle). We then determined the fraction of the EJPs per pyloric cycle that occurred in each bin (Bucher et al., 2006; White and Nusbaum, 2011; Blitz and Nusbaum, 2012).

To determine the decay of the membrane potential after each EJP during VCN-gastric mill and POC-gastric mill rhythms, the difference in membrane potential between each EJP peak and the subsequent trough was measured using Spike2. For a given gastric mill cycle, this difference was normalized by the maximum decay amplitude possible during this cycle (i.e., by the difference between the largest peak EJP membrane potential and the baseline resting potential). The last EJP of each burst was omitted in this analysis because it simply represents the return of the membrane potential to baseline in both versions of the gastric mill rhythm.

The movement output of the gastric mill lateral teeth was quantified by three parameters, all of which were analyzed using Spike2. First, the maximum protraction amplitude was determined by subtracting the value of maximum protraction during the LG burst phase from the value of maximum retraction during the LG interburst phase. The second parameter used for quantification was the period during which the teeth exhibited substantial protraction amplitudes. For this analysis, we defined "substantial protraction amplitude" as the fraction of the burst duration during which the teeth were within $10 \%$ of the maximum protraction amplitude. Similar analyses were performed for measuring substantial muscle tension responses and membrane potential depolarizations. The third parameter used was the total protraction duration of the lateral teeth (see above).

To test whether the pyloric timing of the LG burst (or the corresponding stimulation) was reflected in the tension response of the gm6ab muscle and in the movement of the lateral teeth, tension curves and movement trajectories were searched for turning points. To this end we used an intrinsic analysis function in the Spike2 software to automatically scan the data for all turning points. Turning points indicate local maxima and minima (i.e., where the first derivative equals 0 and a change in sign occurs) and were used as an indication of unsteady tension or movement trajectories. We also compared the slopes of nerve-stimulated tension and movement trajectories measured at the start and at the end of each pyloric-timed pause (i.e., gap) in the LG burst. The approximate duration of these gaps was $200 \mathrm{~ms}$, corresponding to the duration of pyloric pacemaker neuron activity, which indirectly causes the gaps in the LG burst (see below; Blitz and Nusbaum, 2008). Thus, tension and movement slopes were measured in a succession of $40 \mathrm{~ms}$ bins spanning the $200 \mathrm{~ms}$ interval before the onset of each pyloric-timed gap in the LG burst (or stimulation pattern). This period represents the period when LG activity is driving gm6ab contraction and lateral tooth protraction. For comparison, tension and movement slopes were measured for a second set of $40 \mathrm{~ms}$ bins during which the gap in LG activity affected muscle tension and tooth movement, starting at the offset of the gap and ending $200 \mathrm{~ms}$ later. Note that $40 \mathrm{~ms}$ corresponded to the video sampling rate $(25 \mathrm{~Hz})$. All slope values within each $200 \mathrm{~ms}$ interval were then averaged.

For the in vivo analysis, we determined movement trajectories relative to the burst of the pyloric pacemaker neuron PD (pyloric dilator) instead of the LG burstlet, because it was difficult to isolate LG neuron activity in these experiments. We used the PD neuron as a surrogate for $\mathrm{AB}$ (anterior burster) neuron activity because $\mathrm{PD}$ neuron recordings are more accessible. The PD neurons are electrically coupled to $\mathrm{AB}$, and collectively they comprise the pyloric pacemaker ensemble (Marder and $\mathrm{Bu}-$ cher, 2007). The $A B$ neuron synaptically inhibits the projection neurons MCN1 and commissural projection neuron 2 (CPN2), in vitro and in vivo (Blitz and Nusbaum, 2008; Hedrich et al., 2011), and thereby indirectly mediates the pyloric-timed burstlet activity in LG during the POCtriggered gastric mill rhythm. Because the PD burst coincides with the gap in the LG burst, we measured movement slopes during $40 \mathrm{~ms}$ bins for the $200 \mathrm{~ms}$ interval immediately preceding the PD burst and compared them to those occurring immediately after the end of the PD burst. As above, the former of these slope measurements represented the influence of the LG burstlet, while the latter represented the influence of the pyloric-timed gap that occurs in LG between each burstlet. To further demonstrate the pyloric timing of the tooth movement bouts, their cycle period was determined as the time between the peaks of two consecutive bouts. These cycle period values were compared with the corresponding pyloric cycle period, measured via the extracellular $d v n$ or $l v n$ recording as the duration between the onset of two consecutive PD neuron bursts.

Statistical analyses were performed with Excel (version 2003, Microsoft), SigmaStat 3.0 (SPSS), and Matlab (Mathworks). Comparisons were made to determine statistical significance using the paired Student's $t$ test, Mann-Whitney $U$ test or repeated-measures ANOVA unless otherwise indicated. To determine whether the distribution of EJP instantaneous frequencies and EJP decay amplitudes during the POC-gastric mill and VCN-gastric mill rhythms likely corresponded to a single population distribution, we compared them using the two-sample Kolmogorov- 
Smirnov goodness-of-fit hypothesis test (K-S test). In all experiments, the effect of each manipulation was reversible, and there was no significant difference between the premanipulation and postmanipulation groups. Data are expressed as the mean \pm SE, except where noted.

\section{Results \\ Distinct EJP patterns in LG-innervated muscle fibers during the VCN-gastric mill and POC-gastric mill rhythms}

Two distinct albeit interacting rhythmic motor patterns, the gastric mill (chewing) and pyloric (pumping and filtering of chewed food) rhythms, are generated within the STG both in vivo and in the isolated STNS (Marder and Bucher, 2007; Stein, 2009; Hedrich et al., 2011). The gastric mill rhythm is slow (cycle period, $\sim 5-20 \mathrm{~s}$ ) relative to the pyloric rhythm (cycle period, $\sim 0.5-2 \mathrm{~s}$; Fig. 1C). The pyloric rhythm is spontaneously active, in vitro and in vivo, while the gastric mill rhythm is episodic under both conditions, requiring activation by projection neurons that are not spontaneously active.

Two input pathways whose brief $(<2 \mathrm{~min})$ activation triggers long-lasting (10s of minutes) and distinct gastric mill rhythms in the isolated STNS are the mechanosensory VCNs and the peptidergic POC neurons (Beenhakker et al., 2004; Blitz et al., 2008; White and Nusbaum, 2011). Both pathways trigger the gastric mill rhythm by coactivating the same two CoG projection neurons, MCN1 and CPN2 (Beenhakker and Nusbaum, 2004; Blitz et al., 2008). Their ability to trigger distinct gastric mill motor patterns results, at least partly, from their differential regulation of the feedback synapse from the pyloric pacemaker neuron $\mathrm{AB}$ onto these projection neurons, resulting in different MCN1/ CPN2 activity patterns (Blitz and Nusbaum, 2008, 2012). Specifically, during both types of gastric mill rhythms, MCN1 and CPN2 exhibit pyloric-timed bursting during the retraction phase, but their protraction phase activity pattern remains pylorictimed only during the POC rhythm, becoming tonic during the VCN rhythm (Fig. 1C,D).

The VCN-triggered and POC-triggered gastric mill motor patterns are distinct with respect to the burst parameters and relative timing of the activity in several gastric mill motor neurons (White and Nusbaum, 2011). For example, the burst and interburst durations of the protraction motor neuron LG are, on average, longer during the POC-gastric mill rhythm. Most conspicuously, however, the LG neuron exhibits a distinct burst structure during these two rhythms. Specifically, during the POC-triggered rhythm, each LG burst is divided into fast, rhythmic burstlets that are timed by the pyloric rhythm, whereas during the VCN-rhythm the LG burst exhibits a tonic pattern (Fig. $1 C, D)$. LG activity thus reflects the MCN1 and CPN2 burst patterns during VCN and POC protraction (Fig. 1C; Beenhakker and Nusbaum, 2004; Blitz et al., 2008; White and Nusbaum, 2011). Insofar as LG is also a pivotal rhythm-generator and pattern-generator neuron during both of these gastric mill motor patterns (White and Nusbaum, 2011), we aimed to determine whether this distinction in the LG pattern was preserved at the level of the LG-innervated muscles and the resulting protraction movements of the lateral teeth during VCN-driven and POCdriven chewing.

To assess whether these different LG burst structures elicit similarly distinct muscle patterns, we studied the electrophysiological (EJP) and tension responses of muscles that were exclusively innervated by LG (Fig. $1 B$, gm5b, gm6ab, gm8a; Weimann et al., 1991) during the VCN-gastric mill and POC-gastric mill rhythms. Intracellular recordings from individual muscle fibers in gm5b, gm6ab, and gm8a consistently exhibited a resting po- tential of $\sim-70 \mathrm{mV}$ (POC preparations: gm5b, $-73.2 \pm 1.9 \mathrm{mV}$, $n=8$; gm6ab, $-69.3 \pm 1.4 \mathrm{mV}, n=8 ;$ gm8a, $-68.0 \pm 1.1 \mathrm{mV}$, $n=4$; VCN preparations: gm5b, $-72.3 \pm 1.0 \mathrm{mV}, n=8$; gm6ab, $-69.6 \pm 1.4 \mathrm{mV}, n=8$; gm $8 \mathrm{a},-68.8 \pm 1.9 \mathrm{mV}, n=4)$. These values were comparable to previous recordings from these fibers (Stein et al., 2006). Under our experimental conditions, action potentials were not generated in these fibers, as is typical for STNS muscles (Hooper et al., 1986; Weimann et al., 1991; Stein et al., 2006).

Although clear differences in the EJP dynamics between the gm5b, gm6ab, and gm8a muscles were evident (Fig. 2A), the EJP burst pattern matched the LG neuron firing pattern during the VCN-gastric mill and POC-gastric mill rhythms in each muscle type. Specifically, the EJP burst pattern in each muscle type was tonic during the VCN-gastric mill rhythm while it was pyloric rhythm-timed during the POC-gastric mill rhythm (Fig. 2A,B). This distinction was reflected in the distribution of instantaneous EJP rates in the gm6ab muscle during these two gastric mill rhythms (Kolmogorov-Smirnov goodness-of-fit hypothesis test, $p=1.7 \times 10^{-29}, n=6$ for each rhythm; Fig. $\left.2 C\right)$. One clearly distinct region of these distributions during the two rhythms occurred between 2 and $5 \mathrm{~Hz}$ (Fig. 2C). Within this range, there were $\sim 10$-fold more instantaneous EJP frequencies during the POC rhythm than during the VCN rhythm (POC, 273 events; $\mathrm{VCN}, 25$ events), despite the fact that overall there were more total events analyzed during the VCN rhythm (POC, 2225 events, $n=6$; VCN, 2427 events, $n=6$ ).

The $2-5 \mathrm{~Hz}$ range (inter-EJP interval, $0.2-0.5 \mathrm{~s}$ ) represents the range of pyloric rhythm-timed interruptions in LG activity that occur during the POC-gastric mill rhythm (White and Nusbaum, 2011). To determine whether the distinct instantaneous EJP distribution in this range during the POC-gastric mill rhythm likely resulted from the pyloric-timed interruptions in LG activity, we determined the distribution of the LG-mediated EJPs in the LGinnervated muscles relative to the pyloric rhythm. We monitored the pyloric rhythm via extracellular ion recording of the projection neuron MCN1, insofar as MCN1 activity is pyloric-timed during this gastric mill rhythm (Fig. 1C; Blitz et al., 2008; Blitz and Nusbaum, 2012). During the POC-gastric mill motor pattern, the pyloric-timed pattern of LG overlaps with that of MCN1, largely because it is driven by input from MCN1 (Fig. 1C; Wood et al., 2000; White and Nusbaum, 2011; Blitz and Nusbaum, 2012).

As evident in the cumulative data $(n=6)$ in Figure $2 D$, during the POC-gastric mill rhythm, a steady number of LG-mediated EJPs occurred in gm6ab during most of the pyloric cycle, but this number dropped off soon after the end of the MCN1 active period and did not increase again until after MCN1 activity resumed. Thus, the occurrence of LG-mediated EJPs was pyloric rhythm phase-dependent and so these EJPs did indeed exhibit a pyloric-timed pattern during the POC-gastric mill rhythm. We did not perform the comparable analysis during the VCN-gastric mill rhythm because there is no pyloric timing within the LG burst during this motor pattern (Fig. $1 C, D$ ), nor was there any evidence of a rhythmic EJP pattern in gm6ab during the VCN rhythm (Fig. 2B; White and Nusbaum, 2011).

Despite the different EJP patterns, there was no difference in the peak EJP amplitudes during these two rhythms (VCN: $23.5 \pm$ $2.0 \mathrm{mV}, n=6$; POC: $19.7 \pm 4.4 \mathrm{mV}, n=6, p=0.44)$ and, as indicated above, the baseline membrane potential during both rhythms was also the same. However, insofar as the EJPs showed a pyloric-timed pattern after POC stimulation, summation appeared to be periodically interrupted by large amplitude EJP de- 

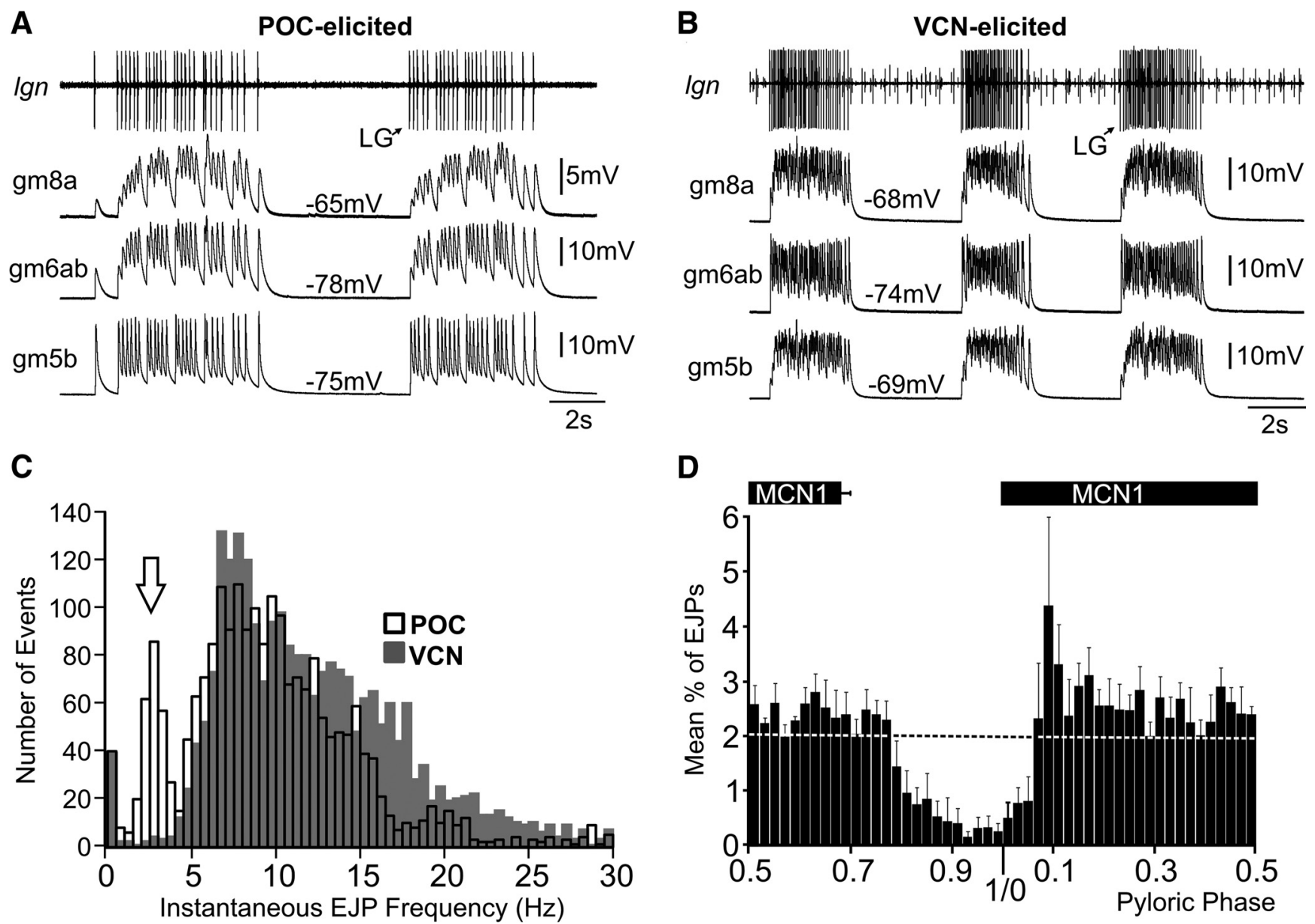

Figure 2. LG-innervated muscle fiber electrical activity patterns replicate the distinct LG neuron patterns during the POC-triggered and VCN-triggered gastric mill rhythms. $A$, Intracellular muscle fiber recordings from three LG-innervated muscles (gm8a, gm6ab, gm5b) show that the electrical activity of these fibers exhibits the same pyloric-timed burst pattern as their innervating motor neuron during the POC-triggered gastric mill rhythm. $\mathrm{LG}$ activity was recorded extracellularly ( $\operatorname{lgn})$. B, Muscle fibers from the same LG-innervated muscles also mimics the $L G$ activity pattern during a VCN-triggered gastric mill rhythm. $\boldsymbol{A}$ and $\boldsymbol{B}$ are from different preparations. C, Distribution of instantaneous EJP frequencies during the POC-triggered (2225 events, $n=6)$ and VCN-triggered gastric mill rhythms ( 2427 events, $n=6$ ). These distributions are highly likely to derive from separate populations (Kolmogorov-Smirnov goodness-of-fit hypothesis test, $p=1.7 \times 10^{-29}$ ). Note the distinct EJP distributions between 2 and $5 \mathrm{~Hz}$ (arrow: POC, 273 events; VCN, 25 events). This region represents the pyloric-timed interruptions in LG activity. The bin width was $0.5 \mathrm{~Hz}$. D, Mean fraction of LG-mediated EJPs per bin in gm6ab muscle fibers during the normalized pyloric cycle across POC-triggered gastric mill rhythms $(n=6)$. The pyloric phase was normalized to the start of the MCN1 burst. The dashed line (mean percentage EJPs, 2\%) represents the overall average percentage of EJPs per pyloric phase when spread evenly throughout the entire normalized pyloric cycle. Note that the $x$-axis begins at phase 0.5 , to center the phase of reduced EJP activity. The bin width was $2 \%$.

cays in this version of the rhythm, which was not the case after VCN stimulation (Fig. $3 A, B$ ). Consequently, during the VCNtriggered EJP bursts, the membrane potential remained within $10 \%$ of the maximum depolarization for considerably longer than during the POC rhythm (VCN: $5.3 \pm 1.4 \%$; POC: $2.5 \pm$ $0.7 \% ; n=6 ; p<0.05 ; t$ test $)$.

We further tested the hypothesis that summation during the POC rhythm was affected by the pyloric-timed gaps in the EJP pattern by comparing the membrane potential before, during, and after the gap. To this end, during the POC rhythm, the minimum membrane potential reached before the last EJP before the gap was compared with that during the gap and the one following the first EJP after the gap. The minimum EJP membrane potential during the gap was more hyperpolarized than that before and after the gap (Pregap, $-61.6 \pm 2.1 \mathrm{mV}$; gap, $-66.2 \pm 2.1 \mathrm{mV}$; Postgap, $-60.4 \pm 2.4 \mathrm{mV} ; n=6, p<0.05 ; F_{(2,10)}=8.31 ; \mathrm{RM}$ ANOVA with Holm-Sidak post hoc comparison).

The influence of the pyloric-timed gaps on the minimum EJP membrane potential during the POC rhythm should also be reflected in the extent to which EJPs decayed toward the baseline resting potential (see Materials and Methods), which should be more pronounced than during the VCN rhythm. We thus normalized EJP decay amplitudes to the decline back to the baseline resting potential of the largest amplitude EJP in a burst (i.e., a value of 1.0 represents the largest EJP decay) and compared the distribution of EJP decays during both rhythms. Figure $3 C$ shows that these distributions represented distinct populations (Kolmogorov-Smirnov goodness-of-fit hypothesis test, $p=2.9 \times$ $10^{-36}, n=6$ fibers for each rhythm), with more large amplitude decays occurring during the POC-rhythm. Specifically, in the region of the normalized EJP decay distribution containing the largest-amplitude decays $(0.6-1.0)$, there were more than twice as many large-amplitude decays during the POC-rhythm (VCN, 328; POC, 761, $n=6$ each; Fig. 3C). This was the case despite the fact that there were more total events analyzed during the VCNrhythm (VCN, 3597 events; POC, 3185 events). Our results thus support the hypothesis that within-burst summation was periodically interrupted by the pyloric-timed pattern of the EJPs. These interruptions during the POC-rhythm could contribute to the 


\section{A POC-elicited}

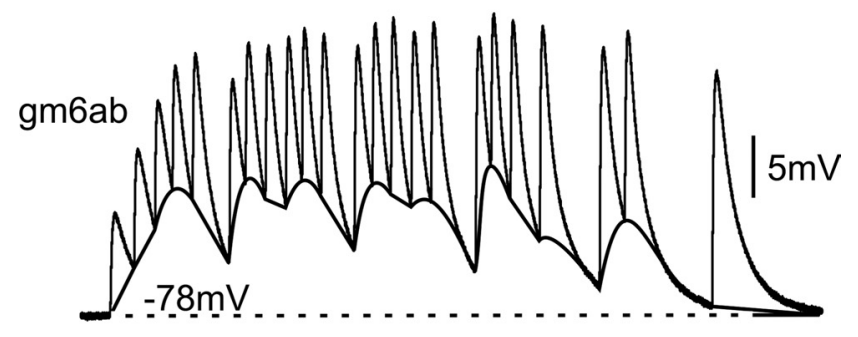

B VCN-elicited
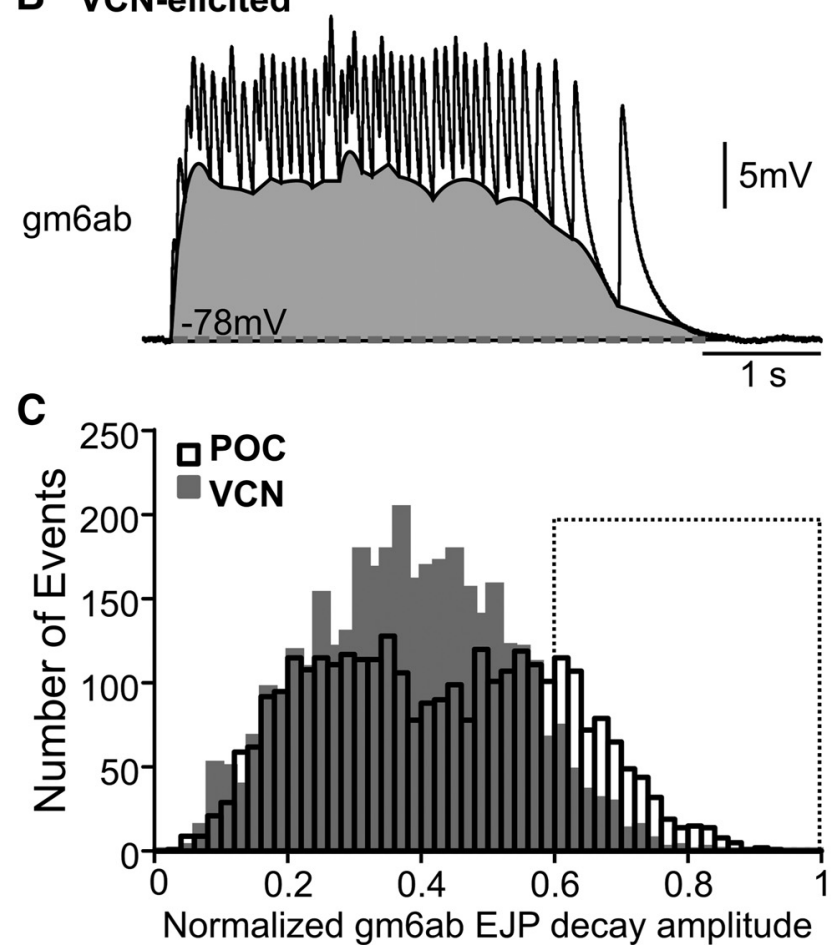

Figure 3. $P O C$-triggered and VCN-triggered gastric mill rhythms elicited distinct distributions of EJP decay amplitudes in the LG-innervated gm6ab muscle. $\boldsymbol{A}, \boldsymbol{B}$, Expanded timescale of one EJP burst in gm6ab during a $(\boldsymbol{A})$ POC-triggered and $(\boldsymbol{B})$ VCN-triggered gastric mill rhythm. Highlighted between the bottom of each burst and the baseline (dashed line) is an approximation of the underlying summation (POC, white; VCN, gray). Note the periodic troughs that were particular to the POC rhythm. C, The EJP decay amplitude distribution in gm6ab muscle fibers during the POC-triggered and VCN-triggered gastric mill rhythms was likely to be derived from distinct populations (Kolmogorov-Smirnov goodness-of-fit hypothesis test, $p=2.9 \times$ $10^{-36} ;$ POC, 3185 events; VCN, 3597 events). Note the larger number of large-amplitude EJP decays (0.6-1.0; boxed area) during the POC rhythm (761 events) relative to the VCN rhythm (328 events). The bin width was 0.02. EJP decays in each burst were normalized to the distance between the largest EJP and the baseline resting potential.

decreased tension generated during this version of the rhythm (see below).

Distinct tension patterns and amplitudes in LG-innervated muscles during realistic VCN-gastric mill and POC-gastric mill rhythm stimulation patterns

Muscle contraction is not only influenced by the muscle fiber membrane potential changes (EJPs) that occur during motor neuron activity, but also by the intrinsic properties of the muscle fibers. In the case of the gastric mill muscles, there are relatively slow contraction and relaxation dynamics (Hooper and Weaver, 2000; Stein et al., 2006). We therefore tested whether the LG- innervated gm6ab muscle contraction faithfully reproduced the pyloric rhythm timing of its EJPs.

We obtained muscle tension measurements in nerve-muscle preparations using the isolated gm6ab muscle driven by realistic stimulation protocols derived from LG activity during POCtriggered and VCN-triggered gastric mill rhythms in the isolated STNS (see Materials and Methods). For each type of gastric mill rhythm, we used a range of rhythms selected from three different preparations to stimulate LG, to ensure that the results would not be dictated by any parameter(s) particular to one specific rhythm. Figure 4 compares the characteristics of each selected motor pattern to the averaged values from previously published studies. Each of these rhythms included parameters that represented rather well the range of rhythms recorded in the previous experiments (Fig. 4B; Beenhakker et al., 2004; White and Nusbaum, 2011). Additionally, all three VCN patterns exhibited tonic LG bursts whereas all 3 POC patterns exhibited pyloric-timed LG burstlets within each burst (Fig. 4A,C).

Similar to the EJP recordings, the within-burst gm6ab tension pattern reflected the LG neuron pattern that drove it (Fig. 5A). Specifically, each VCN-rhythm stimulation pattern consistently elicited a smooth increase in gm6ab tension, followed by a smooth tension decrease after the end of the burst, whereas the tension buildup within each LG burst during the POC-pattern stimulations waxed and waned periodically (each pattern, $n=8$; Fig. 5). Additionally, the periodic dips in tension during each burst of the POC-pattern stimulations appeared to coincide with the pyloric-timed pauses in the LG firing pattern used for these stimulations (Fig. 5B).

To test whether the periodic drops in muscle force within each POC-stimulation burst faithfully represented the pyloric gaps in the LG firing pattern, we first measured the number of (positive plus negative) turning points per burst in the tension trace for all three POC patterns (POC 1, $7.9 \pm 1.1$; POC 2, $8.8 \pm 1.7$; POC 3 , $12.8 \pm 0.8 ; n=8)$. This matched rather well with the number of burstlets (onsets plus offsets) per burst in the LG stimulation pattern (POC 1, 9.6 \pm 0.6; POC 2, $10.8 \pm 1.6$; POC 3, $16 \pm 1.5$ ). The slightly smaller number of turning points than burstlet onsets plus offsets was most likely due to the fact that muscle tension sometimes increased without visible interruption, presumably due to the short duration of some pyloric-timed gaps in the stimulation pattern (Fig. 4A). In comparison, the number of turning points per burst in the force trajectory of the VCN pattern was consistently lower (VCN 1, $2.1 \pm 1.6$; VCN 2, $2 \pm 0$; VCN 3, $2.2 \pm$ 1.6; $p<0.001$, Mann-Whitney $U$ test, $n=8$ ), matching effectively the expected number of 2 (burst start and end) for the VCN patterns.

To further support the hypothesis that the periodic dips in muscle tension during the POC-rhythm stimulations resulted from the pyloric-timed gaps in the LG stimulation pattern, we measured tension velocity at the beginning and end of each LG burstlet (see Materials and Methods). As shown in Figure 6A, the distribution and direction of tension velocities across cycles and preparations was different for the onset and offset of the burstlets $(p<0.001$, Wilcoxon signed rank test, $n=8)$. Specifically, on average the sign of the tension velocity was positive at the start and negative at the end of these burstlets, indicating an increase and decrease of muscle tension, respectively. Insofar as these burstlet onsets and offsets were pyloric rhythm-timed (White and Nusbaum, 2011; Blitz and Nusbaum, 2012), this result supported the hypothesis that the periodic dips in the tension trace resulted from the pyloric timing of the LG stimulation pattern. 
A
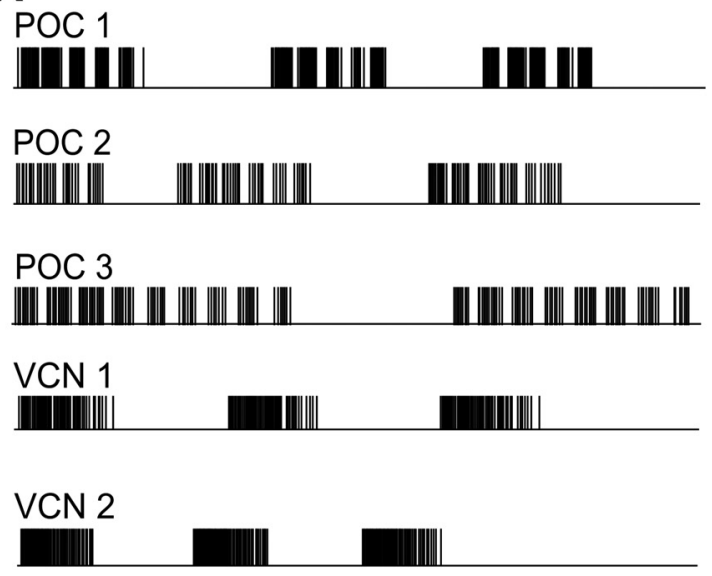

VCN 3

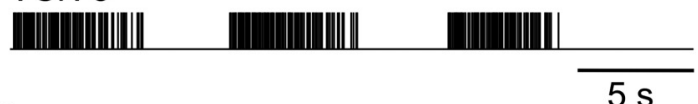

C

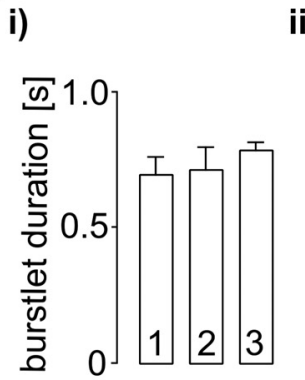

$B_{\text {i) }}$

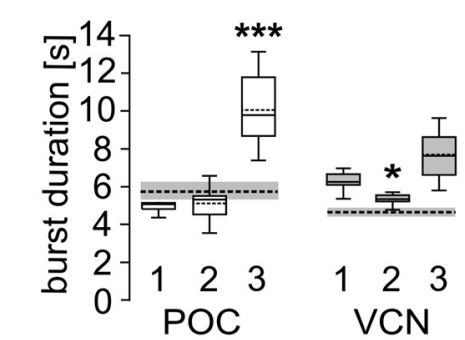

iii)

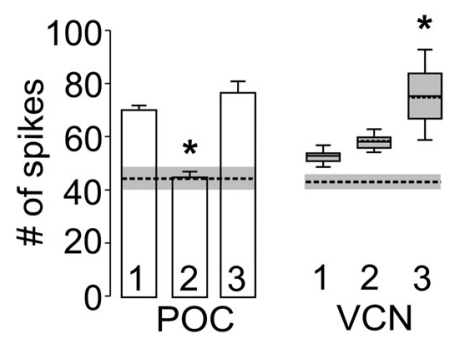

ii)

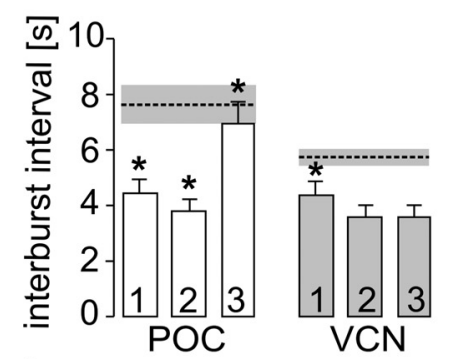

iv)

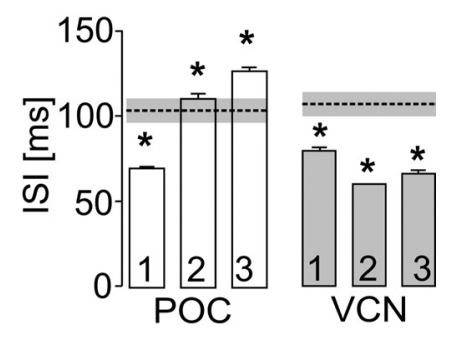

Figure 4. LG neuron burst parameters during the three POC-triggered and three VCN-triggered gastric mill rhythms selected for $L G$ stimulation during recordings of muscle tension and tooth movements. A, Schematic representation of LG-stimulation pulses during the first two or three of the 10 cycles from the three POC-triggered (POC 1-3) and VCN-triggered (VCN 1-3) gastric mill rhythms. Each pattern was derived from a different preparation. $B$, Comparison of mean ( \pm SE) LG neuron (i) burst duration, (ii) interburst interval, (iii) number of spikes per burst, and (iv) interspike interval (ISI) for all six stimulation patterns. Box plots: Whiskers, 5-95 percentiles; boxes, $25-75$ percentiles; median value, continuous line; average, dashed line. Significance $\left({ }^{*} p<0.05 ;{ }^{* * *} p<\right.$ 0.001, Kruskal-Wallis 1-way ANOVA for non-normally distributed data; 1-way ANOVA for normally distributed data) denotes differences from all other rhythms of the same category (POC or VCN rhythms). Dashed lines and associated gray boxes represent the mean values \pm SE, respectively, for a larger set of POC-triggered or VCN-triggered gastric mill rhythms, derived from White and Nusbaum (2011). C, Within-burstlet LG neuron parameters for the three POC patterns. Statistics as in $\boldsymbol{B}$.

For both the VCN and POC stimulation patterns, the rhythmic tension increase consistently relaxed back to baseline before the start of the next stimulation cycle. However, there were consistent differences in both the duration of the substantial tension level and the amplitude of the maximal tension level between these two stimulation patterns. For example, the time during which the tension level during protraction was within $10 \%$ of the maximum was measured and normalized as a percentage of total protraction duration. During VCN stimulation patterns, this percentage (VCN 1, $26.7 \pm 2.7 \%$; VCN 2, $36.4 \pm 3.5 \%$; VCN 3, $31.5 \pm 3.1 \% ; n=8$ each) was approximately 20 -fold larger than during POC stimulation patterns (POC 1, $0.46 \pm 0.3 \%$; POC 2, $0.27 \pm 0.2 \%$; POC 3: $3.87 \pm 1.8 \% ; n=8$ each; $p<0.001$, $F_{(5,37)}=2068.61$; RM ANOVA with Holm-Sidak post hoc comparison; Fig. 6B). Similarly, all three VCN-stimulation patterns elicited large-amplitude maximal tension levels (VCN 1, $0.8 \pm$ $0.1 \mathrm{mN}$; VCN 2, $1.5 \pm 0.2 \mathrm{mN}$; VCN 3, $0.7 \pm 0.1 \mathrm{mN} ; n=8)$ relative to the POC-stimulation patterns (POC 1, $0.26 \pm 0.07$ $\mathrm{mN}$; POC 2, $0.35 \pm 0.04 \mathrm{mN}$; POC 3, $0.33 \pm 0.02 \mathrm{mN} ; n=8$; $p<$ 0.05 each, $F_{(5,37)}=708.91$; RM ANOVA with Holm-Sidak post hoc comparison; Fig. $6 \mathrm{C}$ ). The larger-amplitude tension levels during the VCN-patterns were consistent with the reduced number of large-amplitude EJP decays during the VCN-rhythm (Fig.
3), which might have enabled a sustained elevation of intracellular $\mathrm{Ca}^{2+}$ leading to larger-amplitude tension changes.

Overall, these data indicate that the different LG neuron burst patterns during the VCN-gastric mill and POC-gastric mill rhythms remain distinct at the level of muscle contraction, for at least one LG-innervated muscle.

Distinct lateral teeth chewing movements driven by realistic VCN-gastric mill and POC-gastric mill rhythm patterns

The ability of the gm6ab muscle tension changes to faithfully reproduce its different EJP patterns during the two gastric mill rhythms did not necessarily mean that this distinction would also be present in the tooth movements during chewing. For example, movements occurring in vivo are commonly the consequence of the contraction dynamics of multiple agonist and antagonist muscles, as well as of elastic properties of hinges and ligaments. We therefore assessed the ability of the lateral teeth to protract in VCN-specific and POC-specific patterns when LG was driven by the same realistic stimulation patterns used above to assay gm6ab muscle tension (Figs. 4, 5). For these experiments, we bisected the $d v n$ (Fig. 1A), which contains the LG axon, and stimulated its posterior segment extracellularly with the aforementioned realistic LG patterns while monitoring tooth movement with an en- 

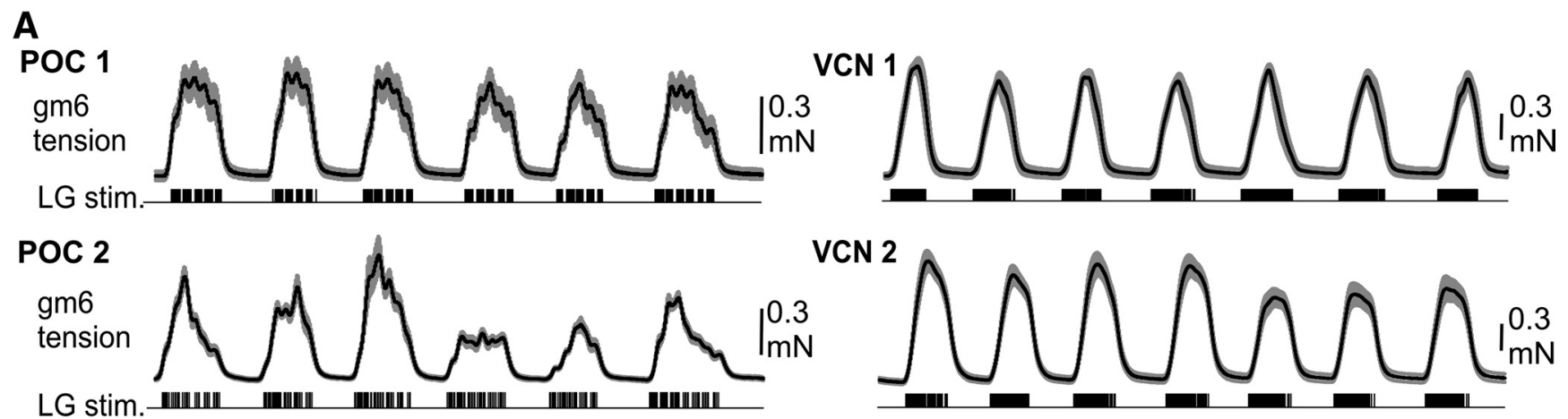

VCN 2
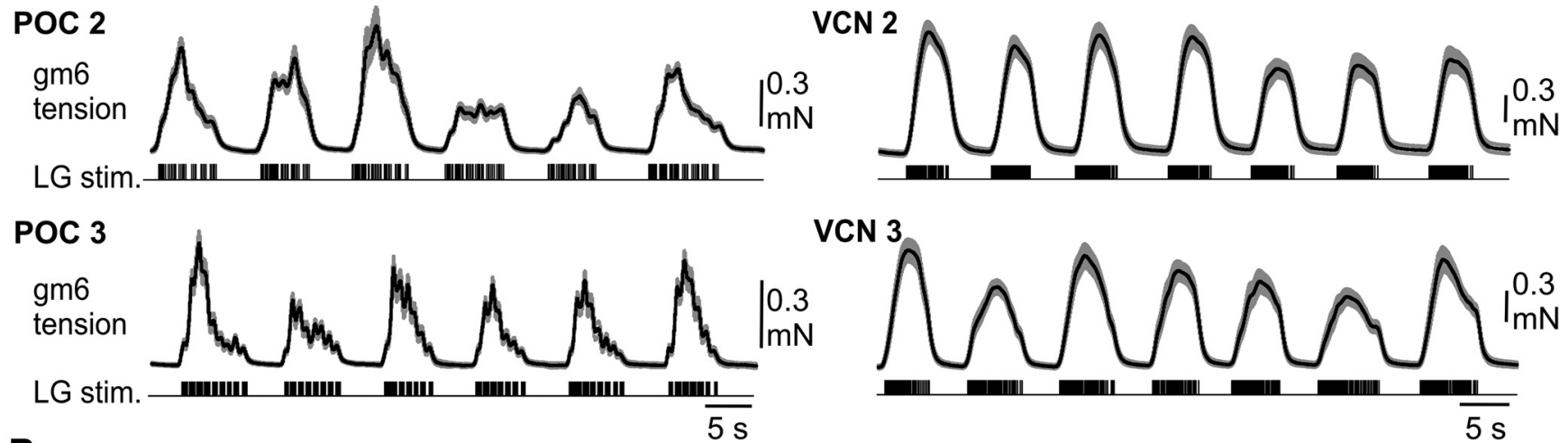

$B$

POC 1

POC 2

VCN 3
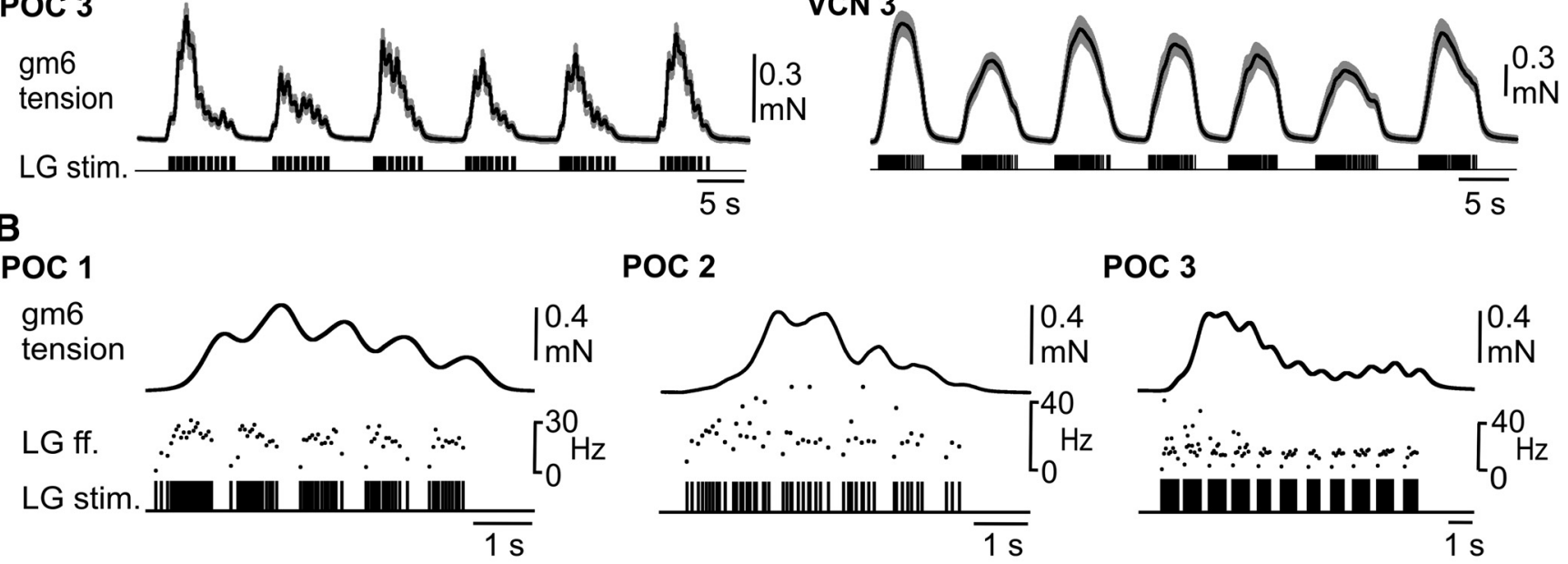

POC 3

VCN 1

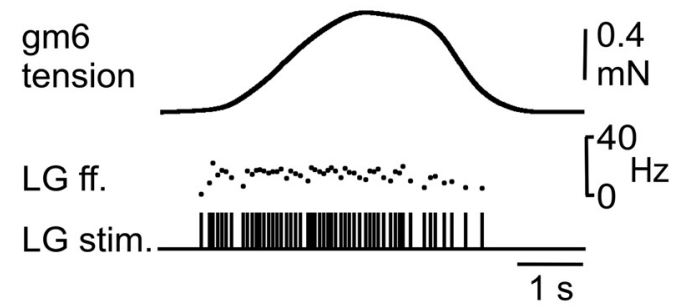

VCN 2

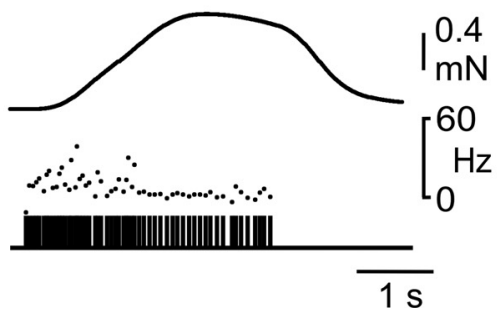

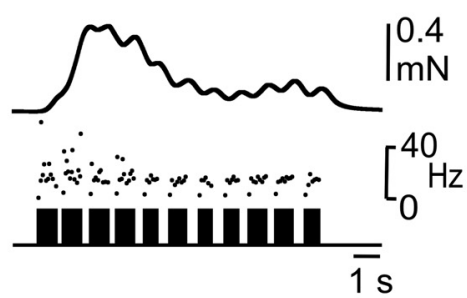

VCN 3

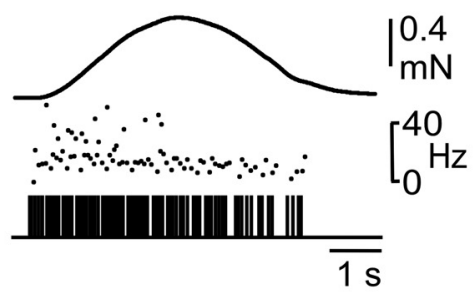

Figure 5. The gm6ab muscle tension faithfully reflected the distinct $L G$ neuron stimulation patterns derived from realistic POC-triggered and VCN-triggered gastric mill rhythms. $A$, Average gm6ab muscle tension in nerve-muscle preparations during the first six LG burst stimulations, using the POC-triggered (POC 1-3,n=8) and VCN-triggered (VCN 1-3, $n=8)$ gastric mill rhythm patterns shown in Figure 4. Black traces represent mean values; gray clouds represent SD. $\boldsymbol{B}$, The gm6ab tension response to LG stimulation during one LG burst of an individual POC 1-3 and VCN $1-3$ gastric mill pattern. The gm6ab tension response tracked the $L G$ stimulation pattern in all examples, including the pyloric-timed tension decays during each POC burst and the smooth tension increase, plateau, and decrease during each VCN burst. All recordings in $\boldsymbol{B}$ are from the same preparation. $L G$ ff. (middle) represents the instantaneous $L G$ stimulation frequency.

doscope ("in situ” experiments; see Materials and Methods). The single LG axon bifurcates at the $d v n / l v n$ junction (Fig. $1 A$ ) and thereby innervates the bilaterally symmetric lateral tooth protractor muscles displayed in Figure $1 B$.

LG neuron activity by itself only drives contractions in the lateral teeth protractor muscles, whereas a coordinated chewing movement involves rhythmic protraction and retraction of the lateral teeth as well as the medial tooth (Heinzel et al., 1993). Without this coordinated pattern, a given movement would not likely express its normal range of motion. We therefore coordinately stimulated the medial tooth protractor (GM) and retractor (DG) motor neurons, using their previously determined activity phases during the POC and VCN rhythms (see Materials and Methods). In so doing, we found the DG neuron also contributed to lateral tooth retraction (data not shown).

Coordinated stimulation of the aforementioned gastric mill motor neurons with realistic VCN-gastric mill and POC-gastric mill rhythm patterns consistently elicited coordinated chewing movements. As is apparent in Figure $7 \mathrm{~A}$, lateral tooth protraction was consistently time-locked to LG stimulation during each gastric mill rhythm pattern ( $n=5$ for each stimulation pattern). Most protractions began with a marked delay after the start of the associated LG burst (Fig. 7B). Similarly, retraction onset was delayed relative to the end of each LG burst.

The VCN-stimulation and POC-stimulation patterns consistently elicited different movement patterns. Specifically, protraction was smooth during each of the three $\mathrm{VCN}$-stimulation patterns, whereas each POC-elicited protraction movement pattern exhibited a series of brief protractions interrupted by brief, small-amplitude decreases or dips in these movements $(n=$ 5; Fig. $7 B$ ). These small-amplitude dips during the POCstimulations appeared to be time-locked to the pyloric rhythmrelated gaps in the LG stimulation pattern. Due to the delayed tooth response, the trough of these dips occurred after the 

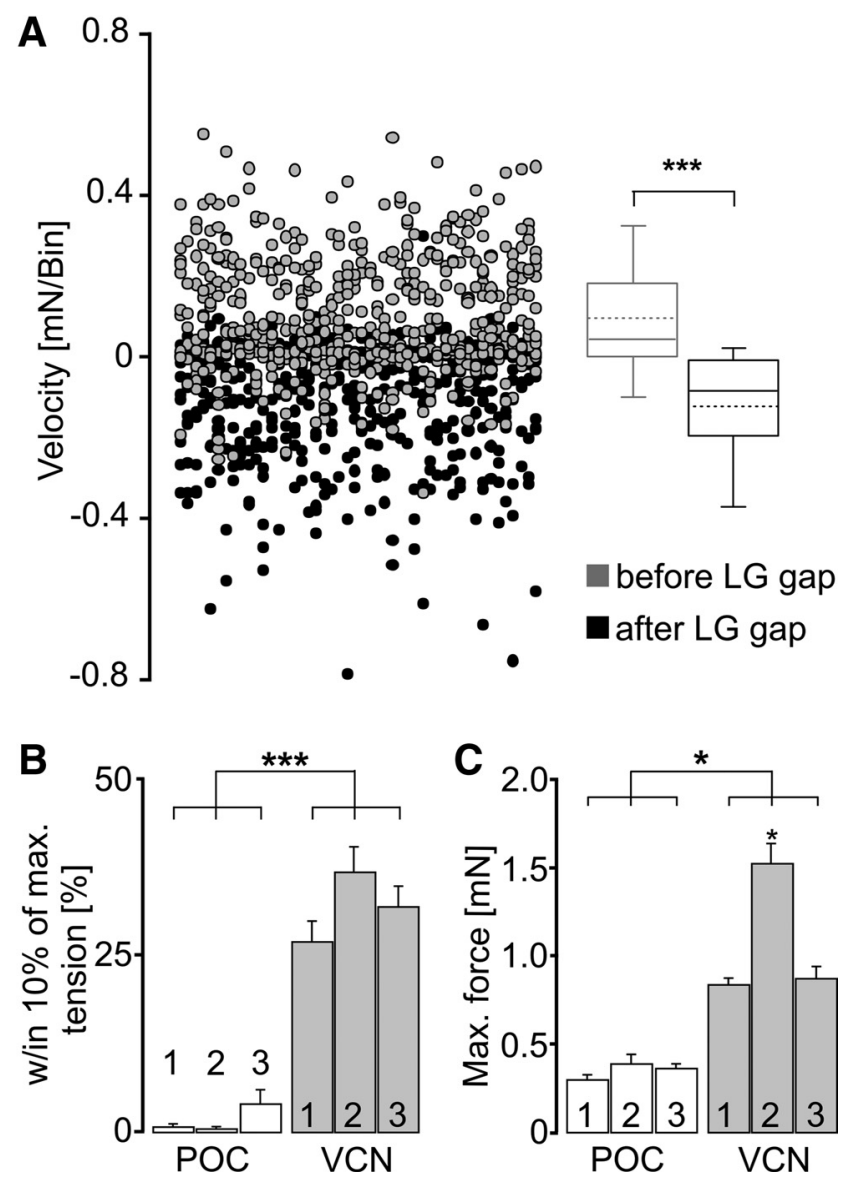

Figure 6. The gm6ab muscle-tension response was distinct when the $L G$ neuron was stimulated using realistic POC-triggered and VCN-triggered gastric mill rhythm patterns. $\boldsymbol{A}$, Distribution of the slopes (i.e., velocity) of the gm6ab tension response (see Materials and Methods) relative to the pyloric-timed LG-stimulation pattern during POC 1-3 pattern stimulations. A total of 672 data points each are plotted for the average tension response ( $40 \mathrm{~ms}$ bin width) within the 200 ms interval before the start of each LG gap, and that within the 200 ms interval after the gap ( $n=8$ preparations). Positive values indicate increasing tension, while negative values indicate decreasing tension. Data points are plotted in ascending order from left to right as obtained from individual measurements within each preparation, with subsequent preparations appended to the right and then wrapped around to start the next row. Data were obtained using all three realistic POC patterns (POC 1-3). Box plots, Whiskers: 5-95 percentiles; boxes, 25-75 percentiles; median, continuous line; average, dashed line $(n=8)$. Tension slopes were different between the two conditions ( ${ }^{* * *} p<0.001$, Wilcoxon signed rank test), exhibiting on average positive slopes before and negative slopes after the gap in the LG burst stimulus. $\boldsymbol{B}$, Substantial gm6ab tension during $\mathrm{LG}$ stimulation occurred for a larger percentage of protraction during VCN patterns than during POC patterns. Substantial tension was defined as the fraction of the burst duration during which tension levels were within $10 \%$ of the maximum tension ( ${ }^{* * *} p<0.001, n=8$, repeated-measures ANOVA). C, Maximum force generated by gm6ab during $L G$ stimulation with VCN and POC patterns. All VCN-stimulus patterns elicited larger force than the POC-stimulus patterns $\left({ }^{*} p<0.05 ; n=8\right.$, repeated-measures ANOVA). The VCN 2 pattern generated a larger force than the VCN 1 and VCN 3 patterns $\left({ }^{*} p<0.05 ; n=8\right.$, repeated-measures ANOVA).

actual LG gaps (i.e., after the start of the subsequent burstlet; Fig. 7B).

To test whether the dips in the POC-driven lateral tooth protraction movements indeed corresponded to the pyloric-timed gaps in the LG stimulation pattern, we examined the protraction movement trajectories for turning points, analogous to the analysis we performed for muscle tension (see above). For each POCstimulation pattern, the number of turning points (positive plus negative) per protraction phase was determined and compared with the number of burstlets (start plus end per burstlet) per stimulated burst. The mean number of turning points depended on the POC variant (POC 1, 8.4 \pm 1.2 ; POC 2, $6.3 \pm 1.1$; POC 3 , $11.6 \pm 1.0)$, and in general corresponded to the number of $L G$ burstlets per variant (POC 1, 9.6 \pm 0.5 ; POC 2, $10.8 \pm 1.6$; POC $3,16 \pm 1.5)$. In each case, however, they were lower than the mean number of burstlets, and were also lower than the equivalent turning point values obtained in the muscle-tension experiments. One likely reason for this discrepancy was that the initial burstlets in a burst sometimes possessed relatively short interburstlet intervals (Fig. 4A), which resulted in a steep increase in tooth protraction and no apparent dip in the movement.

Unlike the case for the POC stimulations, the number of (positive plus negative) turning points in the lateral tooth protraction movement trajectory during the VCN pattern stimulations (VCN $1,2.3 \pm 0.4 ; \mathrm{VCN} 2,2.2 \pm 0.3 ; \mathrm{VCN} 3,2.5 \pm 0.2)$ matched very well the fact that there were no pyloric-timed interruptions in the LG burst during these stimulations (i.e., the expected value was 2 ). The number of turning points in the tooth protraction movement elicited by VCN-pattern stimulation was also significantly lower than during the POC-pattern stimulations $(n=5, p<$ $0.001, F_{(5,30)}=105.98$, repeated-measures ANOVA, Holm-Sidak post hoc comparison).

To test whether the dips in tooth protraction movement did correspond to the pyloric-timed gaps of the LG stimulation, we measured the slope of the movement trajectory (i.e., movement velocity) and determined it for an averaged $40 \mathrm{~ms}$ bin within the $200 \mathrm{~ms}$ before the onset of a LG burstlet gap and compared it to the slope after the end of the gap (Fig. $8 A$ ), as we did for the gm6ab muscle-tension responses in Figure $6 A$. Our expectation, based on the hypothesis that the periodic protraction dips resulted from the gaps in the LG-stimulation pattern, was that there would be a net positive slope (i.e., protraction) in the movement trajectory before the start of each gap and a net negative slope (i.e., dip) after it. These movement velocities lag relative to the LG activity that drives them (Figs. $7 B, 8 B$ ). Movement velocities were indeed different during those two conditions $(p<0.05$, Wilcoxon signed rank test, $n=5$; Fig. $8 A$ ). Movement velocities were generally positive (median, $0.07 \mathrm{MT} / \mathrm{bin} ; 25 \%$ quartile, 0.0006 MT/bin; 75\% quartile, $0.11 \mathrm{MT} / \mathrm{bin}$ ) during the $200 \mathrm{~ms}$ before each gap, while they were negative immediately after the gap (median, $-0.11 \mathrm{MT} / \mathrm{bin} ; 25 \%$ quartile, $-0.19 \mathrm{MT} / \mathrm{bin} ; 75 \%$ quartile: $-0.05 \mathrm{MT} / \mathrm{bin})$. Overall, only $26 \%$ of the positive slope velocities occurred after the gap, while $95 \%$ of the negative velocities occurred at these times $(n=5)$.

As shown in Figure $8 B$, both types of realistic gastric mill rhythm-related LG-stimulation patterns enabled the lateral teeth to meet at the midline of the gastric mill compartment during protraction, albeit with differences in the associated parameters of these movements. For example, the VCN-type LG-stimulation patterns enabled the lateral teeth to consistently meet at the midline during each protraction cycle $[n=150$ cycles $(30$ cycles/crab, 5 crabs)]. On average, the teeth remained within $10 \%$ of the maximum protraction amplitude for $\sim 50 \%$ of the LG burst duration (VCN 1, $51.3 \pm 4.2 \%$; VCN 2, $47.2 \pm 5 \%$; VCN 3, $50.9 \pm$ $3.8 \% ; n=5$ each; Fig. $8 C$ ). In contrast, the teeth did not always reach the midline during $\mathrm{POC}$ stimulation, consistent with the smaller maximal force generated by the gm6ab muscle during POC rhythms (Fig. 6C). On average, the midline was reached by both lateral teeth in only $51 \%$ of the POC-driven cycles (POC 1 , $n=24$ of 47 cycles; POC $2, n=23$ of 45 cycles; POC $3, n=37$ of 78 cycles; $n=5$ each). Additionally, during POC-driven movements, the teeth spent a considerably smaller fraction of the burst duration $(\sim 29 \%)$ within $10 \%$ of the maximum protraction am- 


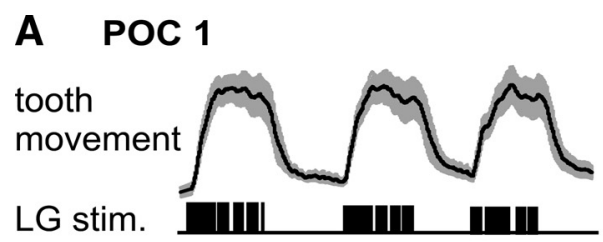

VCN 1

POC 2

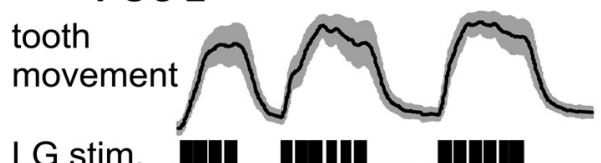

VCN 2
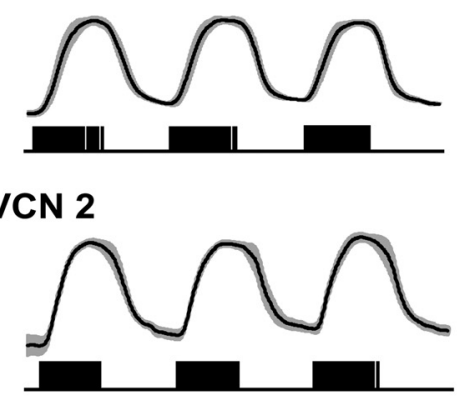

POC 3

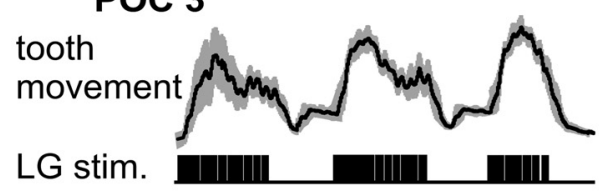

VCN 3

B

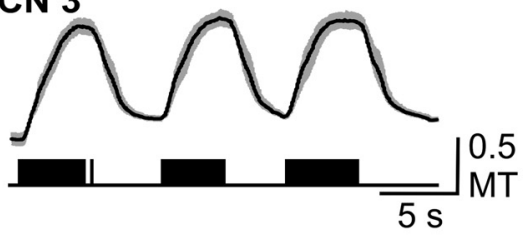

i) $\operatorname{POC} 1$

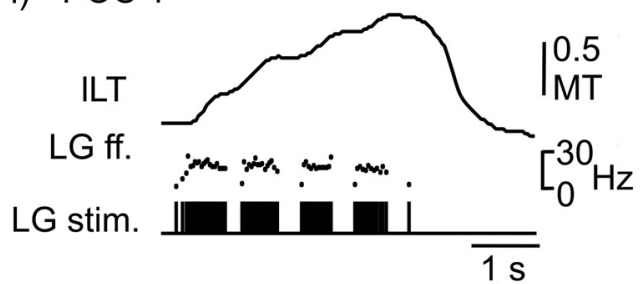

ii) $\mathrm{POC} 2$

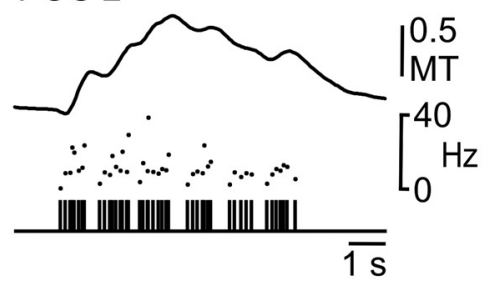

iii) POC 3

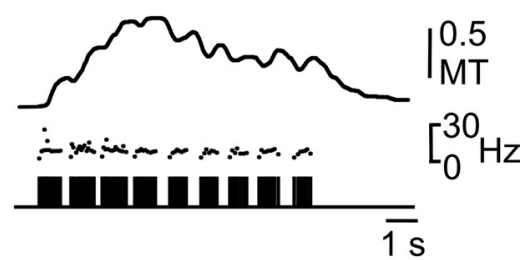

vi) $\operatorname{VCN} 3$

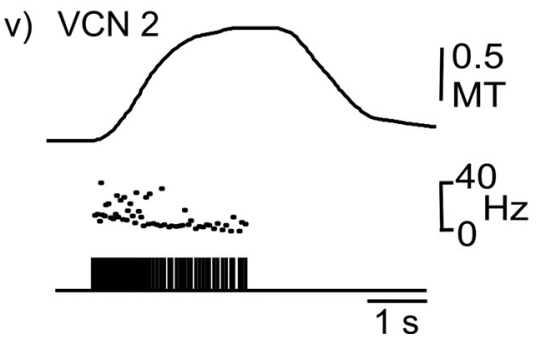

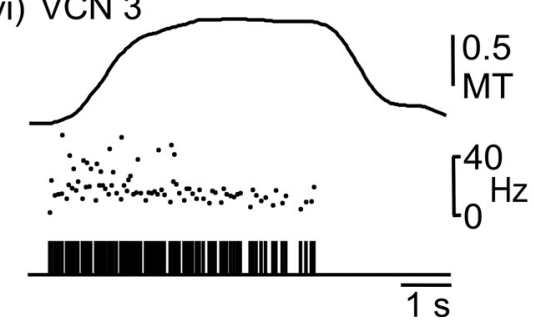

Figure 7. Lateral tooth protraction movements in situ faithfully reflected the distinct $L G$ neuron stimulation patterns derived from realistic POC-triggered and VCN-triggered gastric mill rhythms. $A$, Population data ( $n=5$; black lines, mean values; gray clouds, SD) for lateral tooth movements resulting from LG-stimulation patterns using the same realistic POC-triggered (POC 1-3) and VCN-triggered (VCN 1-3) gastric mill rhythms used for gm6ab tension experiments (Fig. 4). Data were obtained from endoscope video recordings in crabs wherein the dvn was bisected and stimulated via a hook electrode to drive LG activity (see Materials and Methods). Three (of 10) successive LG-stimulus trains and the corresponding lateral tooth movements are shown for each of the POC and VCN stimulus patterns. Tooth movement was normalized to the widest point of the medial tooth (MT). B, Individual examples of lateral tooth protraction movements from single LG-stimulated bursts during each of the POC and VCN rhythms, shown at an expanded time scale. Note the dips in the protraction movements that correlate with the pyloric-timed silent periods in the LG-stimulation patterns during the three POC rhythms, in contrast to the smooth protraction movements during the three VCN rhythms. All recordings in $B$ were from the same preparation. LG ff. represents the instantaneous stimulation frequency used for activating $L G$.

plitude (POC 1, $27.4 \pm 12.3 \%$; POC 2, $31.6 \pm 8.6 \%$; POC 3, $27.4 \pm 6.6 \% ; n=5, p<0.001, F_{(5,30)}=904.4$; repeated-measures ANOVA with Holm-Sidak post hoc comparison; Fig. $8 C$ ). These briefer durations were reminiscent of the brief duration during which tension amplitudes were substantial in gm6ab during these same LG stimulation protocols (Fig. 6B).

Despite the fact that only the VCN-stimulated patterns moved the lateral teeth all the way to the midline in every cycle, the average maximal protraction amplitude was equivalent across all six stimulation patterns used (3 POC patterns, $3 \mathrm{VCN}$ patterns: $p=0.18$ for all comparisons, $n=5, F_{(5,30)}=9.75$; repeatedmeasures ANOVA with Holm-Sidak post hoc comparison; Fig. $8 D)$. This result contrasted with the considerably larger average maximal gm6ab muscle-tension amplitude during the VCNstimulation patterns (Fig. 6C). This discrepancy might have re- sulted from an underestimate of the full potential for each protraction movement because, during chewing, each lateral tooth is prevented from moving past the midline due to its contact with the other lateral tooth.

Together, these data support the hypothesis that the lateral tooth protraction movement pattern is driven by the LG neuron burst pattern, and that these movement patterns are distinct during the VCN-triggered and POC-triggered gastric mill rhythms.

Distinct lateral teeth chewing movement patterns result from stimulating the cardiac gutter (VCN) and POC pathway in vivo In the preceding experiments, lateral teeth movements were monitored when their innervating protraction motor neuron (LG) was stimulated directly using different patterns in an open loop system. We next aimed to determine (1) whether distinct 
VCN-triggered and POC-triggered gastric mill activity patterns of the LG neuron indeed occur in the fully intact crab, where the gastric mill circuit is influenced by sensory feedback, circulating hormones, and parallel descending inputs (Marder and Bucher, 2007; Stein, 2009; Blitz and Nusbaum, 2011) and, if so, whether (2) such patterns still drive distinct lateral teeth protraction movements. To this end, we triggered the $\mathrm{VCN}$ gastric mill rhythm by mechanical stimulation of the cardiac gutter in the cardiac sac stomach compartment (see Materials and Methods; Beenhakker et al., 2004), and we triggered the POC gastric mill rhythm by extracellular stimulation of the $c o c$, as done in vitro (see Materials and Methods; Blitz et al., 2008).

Brief mechanical stimulation (duration, $\sim 2-15 \mathrm{~s}$ ) of the cardiac gutter in vivo triggered a VCN gastric mill rhythm that persisted for $2-20 \mathrm{~min}(n=11$; Fig. $9 A)$, similar to previous cardiac gutter stimulations in reduced preparations (Beenhakker et al., 2004). On average, during the cardiac gutter-triggered gastric mill rhythms, the gastric mill cycle period $(11.33 \pm 4.6 \mathrm{~s}, n=11)$ was similar to that during in vitro $\mathrm{VCN}$ gastric mill rhythms ( 10.8 s; Beenhakker et al., 2004). Despite the similarity in the cycle period in vivo and in vitro, the protraction duration in vivo was considerably briefer (LG burst: $2.58 \pm 0.7$ s, $n=11$; duty cycle: $23 \%$ in vivo vs $57 \%$ in vitro) while the retraction duration was prolonged (LG interburst: $8.77 \pm 4.6 \mathrm{~s}, n=11$; duty cycle: $77 \%$ in vivo vs $43 \%$ in vitro; Beenhakker et al., 2004). Analogous to the LG burst duration in vivo, the LG-elicited protraction of the gastric mill teeth was also brief $(2.61 \pm$ $0.5 \mathrm{~s}, n=11)$. In contrast, the POCtriggered gastric mill rhythms (Fig. 9B) in vivo were fast (cycle period: $8.05 \pm 2.3 \mathrm{~s}$, $n=8)$ relative to those in vitro $(\sim 13.4 \mathrm{~s}$; Blitz et al., 2008; White and Nusbaum, 2011) with both the LG burst duration $(4.24 \pm 1.8 \mathrm{~s})$ and interburst duration $(3.74 \pm 1.6 \mathrm{~s})$ being briefer than the comparable events in vitro. Lateral tooth protraction duration during these POC rhythms was similar to the LG burst duration that drove it $(4.57 \pm 0.8 \mathrm{~s}, n=8)$.

During the cardiac gutter (VCN)-triggered gastric mill rhythms, each lateral tooth protraction event was a reasonably smooth, continuous movement (Fig. 9A). These protraction movements consistently resulted in the two lateral teeth coming together at the midpoint of the gastric mill compartment (fraction of cycles: $96.1 \pm 1.8 \%, n=11$ ) as also occurred during the imposed VCN patterns. On average, the teeth remained in the fully protracted position for a duration $(1.32 \pm 0.1 \mathrm{~s} ; n=11)$ that corresponded to $49.6 \pm 3.8 \%$ of the protraction duration of the
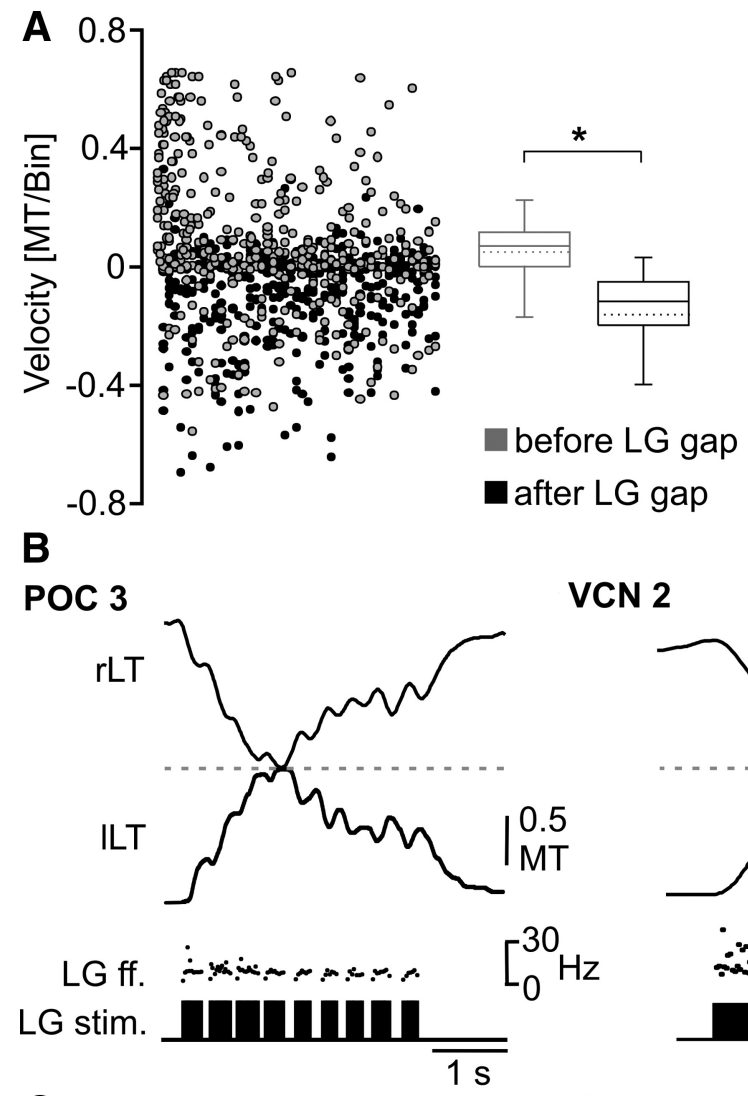

VCN 2
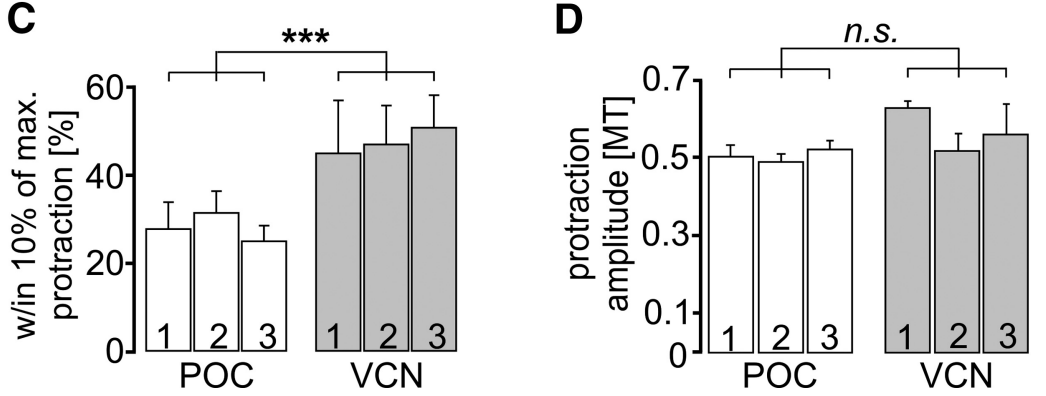

Figure 8. The lateral tooth protraction movement in situ was distinct when the $L G$ neuron was stimulated using realistic $P O C$ and VCN gastric mill rhythm patterns. $A$, Distribution of tooth movement slopes (i.e., velocities) relative to the pyloric-timed $L G$ burstlet pattern during realistic $\mathrm{POC}$ pattern (POC 1-3) stimulation. Movement velocities were significantly different between the two conditions ( ${ }^{*} p<0.05$, Wilcoxon signed rank test, $n=5$ ), with the average velocity of the movement response ( 40 ms bin width) within the $200 \mathrm{~ms}$ interval before the start of each LG gap being positive, and that within the $200 \mathrm{~ms}$ interval after the gap being negative. Data points include 459 events for each condition $(n=5)$. Box plots as in Figure 6. $\boldsymbol{B}$, Coordinated movement of the two lateral teeth, obtained from endoscope video analysis (see Materials and Methods), during a LG-stimulated burst from realistic POC-triggered and VCN-triggered gastric mill rhythms. Note the different protraction patterns, which reflect the different $L G$ stimulation patterns ( $\mathrm{LG}$ stim., LG ff.), as well as the fact that the lateral teeth meet at the midline of the gastric mill compartment (dashed line) for a longer duration during the VCN-stimulation pattern. rLT, Right lateral tooth; ILT, left lateral tooth. C,D, The percentage of each protraction duration during which the teeth were within $10 \%$ of the maximum protraction was larger during stimulation with VCN patterns ( ${ }^{* * *} p<0.001, n=5$, repeated-measures ANOVA), but the protraction amplitude during the POC-stimulation and VCN-stimulation patterns were equivalent ( $p=0.18, n=5$, repeated-measures ANOVA).

lateral teeth, comparable to the value obtained from the in situ experiments above $(\sim 50 \%)$. There was no obvious evidence for any fast, pyloric-timed rhythmic movements during any individual VCN-protraction phase. There were, however, pyloric-timed retraction movements in a subset of these experiments $(n=4$ of 11; Fig. 9A).

In contrast to the VCN-triggered gastric mill rhythms in vivo, the lateral teeth protraction movements during the $\operatorname{coc}$ (POC)triggered gastric mill rhythms in vivo were consistently divided into a series of relatively brief, rhythmic movements $(n=8$; Fig. 


\section{A Cardiac Gutter Stimulation}

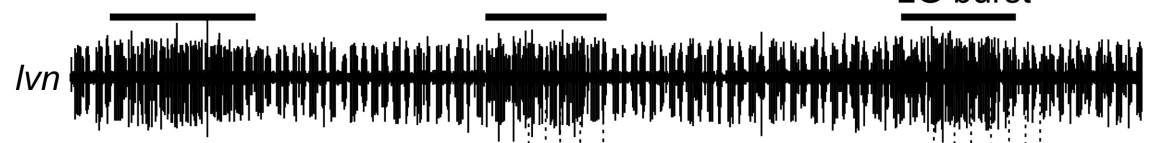

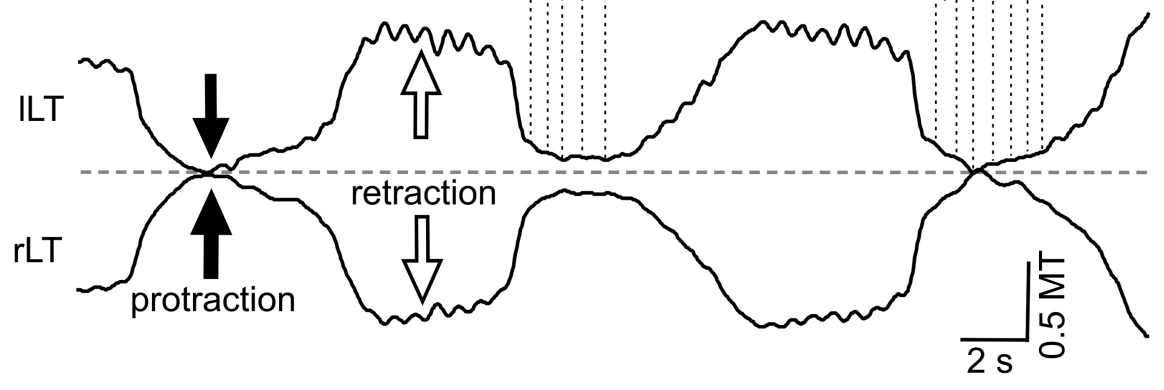

B COC Stimulation
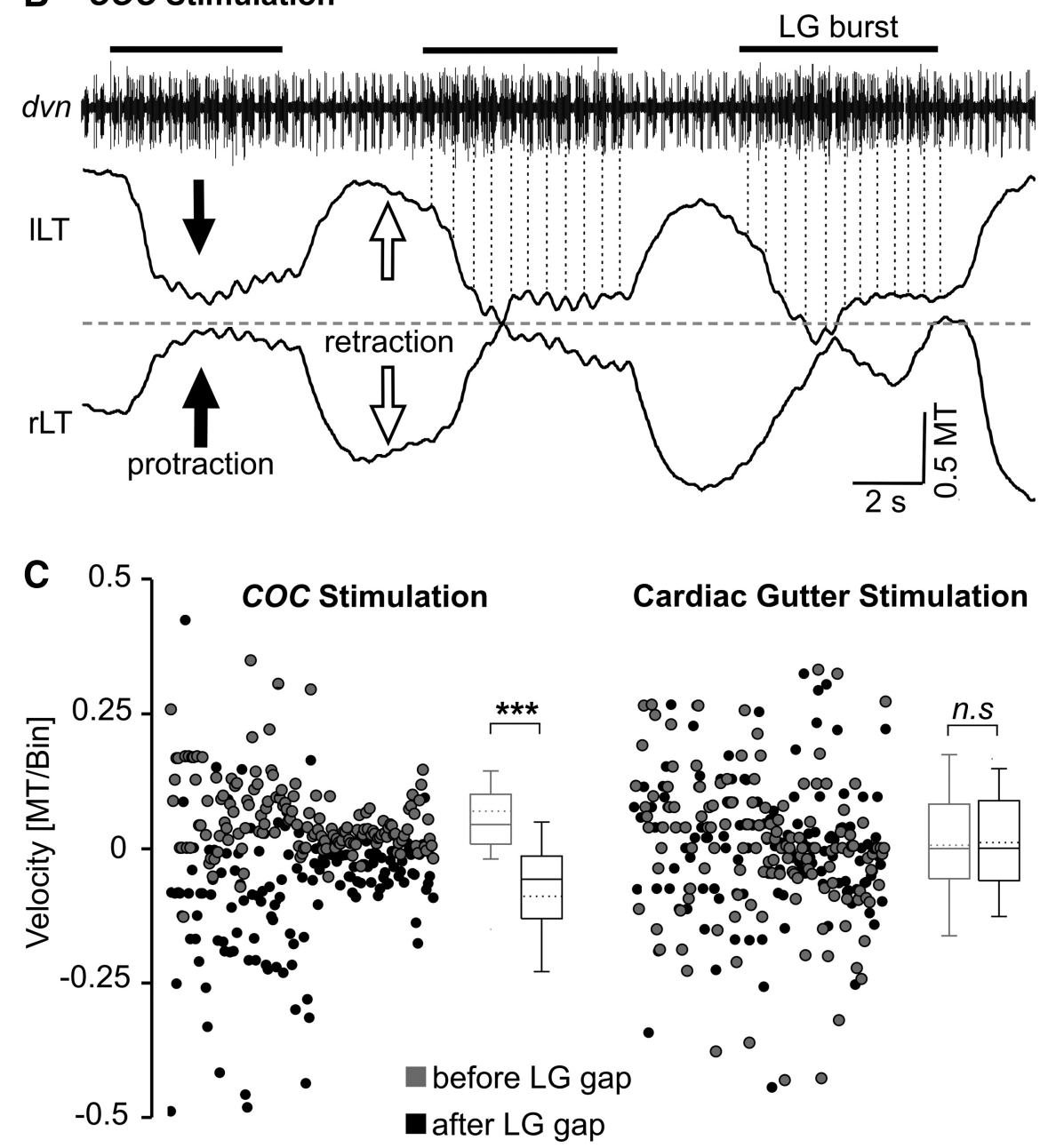

Figure 9. Lateral tooth protraction movements exhibited pyloric timing during coc-triggered (POC) but not cardiac guttertriggered (VCN) gastric mill rhythms in vivo. A, Original recording of a gastric mill rhythm ( $/ v n)$ and the associated lateral tooth movements after mechanical stimulation of the cardiac gutter. Note the relatively smooth protraction movements that lack pyloric timing. Dotted vertical lines connect the pyloric rhythm-timed PD neuron bursts ( $/ v n)$ to the left lateral tooth protraction movements. Pyloric-timed movements were clearly present during retraction. The pyloric rhythm (cycle period, $\sim 0.85 \mathrm{~s}$ ) was recorded as a series of relatively brief bursts in two units (larger unit, LP neuron; smaller unit, PD neuron), most evident during the LG interbursts. LG neuron action potentials were approximately the same amplitude as those of LP and are thus not readily distinguished from them when LP is active. Dashed line, Midline of the gastric mill compartment; black bars, LG neuron bursts; ILT, left lateral tooth; rLT, right lateral tooth. Movements were normalized to the width of the medial tooth (MT). $\boldsymbol{B}$, Original recording of a gastric mill rhythm after coc stimulation. LG neuron activity $(d v n)$ is pyloric rhythm timed, overlapping with and approximately equal in amplitude to that of the pyloric LP neuron (largest units). During protraction, these coactive units exhibit long bursts, due to the $L G$ neuron activity (black bars), relative to retraction when only LP is active. Note the pyloric timing of the protraction movements (dotted lines), and the lack of such timing during retraction. C, Comparison of lateral tooth movement velocities (i.e.
$9 B)$. These fast rhythmic movements were reminiscent of the pyloric-timed LG activity during POC rhythms in the isolated STNS, as well as gm6ab tension increases and lateral tooth protraction movements during imposed POC rhythms (Figs. 1C, $5,7,8)$. Within each protraction episode, these fast rhythmic movements occurred with a mean interval $(0.67 \pm 0.2 s ; n=8)$ that was within the normal range for the pyloric rhythm, both in vitro and in vivo (Heinzel et al., 1993; Beenhakker et al., 2004). In the subset of these experiments that included an extracellular recording of the pyloric rhythm (Fig. 9B), the pyloric cycle period was $0.85 \pm 0.6 \mathrm{~s}(n=4)$ during the POC-triggered gastric mill rhythm. This value corresponded well with the mean cycle period of the fast rhythmic tooth movements during protraction in these same experiments $(0.86 \pm 0.3 \mathrm{~s}, n=4)$. The teeth remained in the fully protracted position for $0.34 \pm$ $0.05 \mathrm{~s}$ (percentage of protraction duration: $7 \%, n=8$ ), similar to the values obtained above in the in situ experiments. As was the case for the tooth movements driven by realistic LG-stimulation patterns, the duration of the fully protracted position was significantly shorter than during the VCN-triggered gastric mill rhythms ( $p<0.001$, unpaired $t$ test, $n=8$ for POC, $n=11$ for VCN). Although LG neuron activity could not be distinguished from that of the lateral pyloric (LP) neuron during these POC-induced gastric mill rhythms, there were clear pylorictimed gaps in the combined LG/LP neuron activity $(n=4$; Fig. $9 B)$. Retraction during these POC-induced gastric mill rhythms was consistently smooth (i.e., not pyloric-timed; $n=8$; Fig. $9 B$ ).

To determine whether the periodic changes observed in the trajectory of the lateral tooth protraction movements after $\operatorname{coc}$ (POC) stimulation were indeed

slope of movement, see Materials and Methods) in vivo, measured and averaged from $40 \mathrm{~ms}$ bins within an interval of 200 $\mathrm{ms}$ before the start of the PD burst and a comparable interval after the end of the PD burst, during gastric mill rhythms triggered by coc stimulation (left) and cardiac gutter stimulation (right). Box plots as in Figures 6 and 8. During coc-triggered rhythms, the average movement velocity was positive when measured before the PD burst, while it was negative after the PD burst ended ( $p<0.001, n=8$, Mann-Whitney $U$ test). There was no difference between these two time points during cardiac gutter-triggered rhythms (n.s., not significantly different; $p=0.76, n=8, t$ test). Note that, while the data from the cardiac gutter-triggered rhythms were normally distributed, we displayed the data distribution as box plots for comparison to the coc-triggered rhythms. 

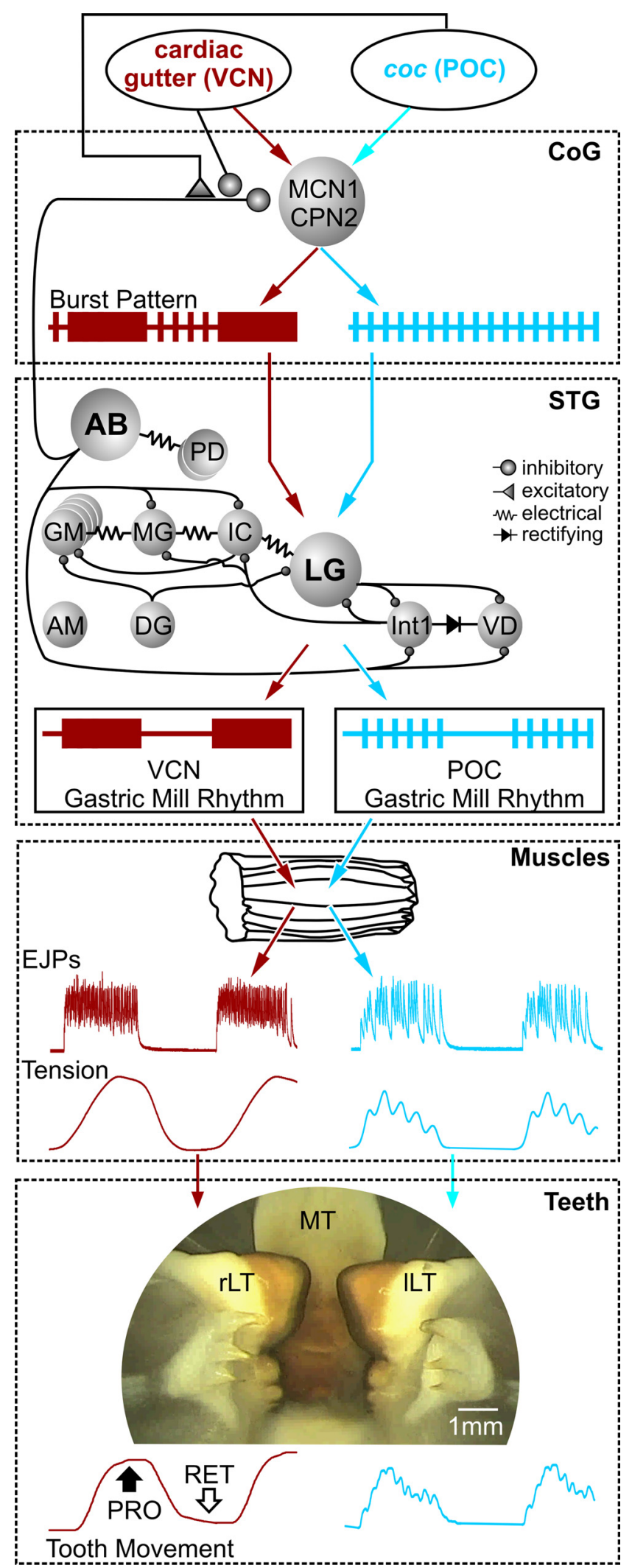

Figure 10. The distinct gastric mill motor patterns elicited in the isolated STNS by different extrinsic inputs drive comparably distinct patterns of EJPs and muscle tension in semi-intact preparations and lateral tooth protraction movements in vivo. In the isolated STNS, stimulating the VCN or POC pathways triggers long-lasting activation of the same two projection neurons (MCN1, CPN2), which then exhibit VCN-specific and POC-specific burst patterns (shown schematically; $(0 G)$. These different patterns result at least partly from differential presynaptic regulation of $A B$ neuron feedback to these projection neurons. The distinct projection neuron timed-locked to the pyloric rhythm, while there was truly no pyloric influence during the cardiac sac (VCN)-triggered protraction movements, we measured the slope of the protraction movement trajectory (i.e., movement velocity) during both rhythms. These measurements were made both $200 \mathrm{~ms}$ before each PD burst onset (i.e., before the onset of a pyloric-timed gap in the LG burst) and $200 \mathrm{~ms}$ after the end of the PD burst. These time points corresponded to the ones we used to measure protraction movements during LG stimulation in situ with the three realistic POC-rhythm patterns (Fig. 8A).

During the POC rhythm there were distinct distributions of positive and negative velocities at the two measured time points $(p<0.001$, Mann-Whitney $U$ test, $n=8$; Fig. 9C). The teeth movements during the POC rhythms exhibited mostly positive movement velocities (median, $0.04 \mathrm{MT} / \mathrm{bin} ; 25 \%$ quartile, 0.01 MT/bin; 75\% quartile, $0.11 \mathrm{MT} / \mathrm{bin}$ ) during the $200 \mathrm{~ms}$ before each gap, while the velocities were mostly negative immediately after the gap (median, $-0.06 \mathrm{MT} / \mathrm{bin} ; 25 \%$ quartile, $-0.13 \mathrm{MT} /$ bin; $75 \%$ quartile, $-0.01 \mathrm{MT} / \mathrm{bin})$. Only $13 \%$ of the positive velocities (which protract the tooth) occurred after the PD burst, while $95 \%$ of the negative velocities (retracting the tooth) occurred at these times. In contrast, during the VCN rhythm, there was no difference in the velocity distribution between the time point before each gap (median, $0 \mathrm{MT} / \mathrm{bin} ; 25 \%$ quartile, -0.05 MT/bin; 75\% quartile, $0.08 \mathrm{MT} / \mathrm{bin}$ ) and after each gap (median, $0 \mathrm{MT} / \mathrm{bin} ; 25 \%$ quartile, $-0.06 \mathrm{MT} / \mathrm{bin} ; 75 \%$ quartile, $0.09 \mathrm{MT} /$ bin; $p=0.76, t$ test, $n=8$ ).

\section{Discussion}

In this paper we show, in an identified motor neuron, that the different action potential burst structures which help distinguish two versions of a rhythmic motor pattern in the isolated nervous system, drive comparably distinct patterns in the muscles they innervate and in the associated behavior (Fig. 10). Specifically, in the isolated STNS, the LG protractor neuron burst exhibits a tonic pattern during the VCN-triggered gastric mill rhythm, whereas during the POC-triggered gastric mill rhythm the LG burst is divided into a series of brief burstlets (White and Nusbaum, 2011). Here we have shown that these distinct LG burst patterns are reliably transferred to LG-innervated muscles at the level of both EJPs and tension, and to the resulting lateral tooth protraction movements during chewing (Fig. 10).

There is an extensive literature establishing that neuromodulation enables individual identified CPGs to generate distinct motor patterns in the isolated nervous system (Marder and Calabrese, 1996; Marder and Bucher, 2001; Marder et al., 2005; Dickinson, 2006; Doi and Ramirez, 2008; Stein, 2009; Berkowitz et al., 2010; Harris-Warrick, 2011; Marder, 2012; Nusbaum and Blitz,

$\leftarrow$

activity patterns drive different gastric mill motor patterns from the gastric mill CPG, including different burst patterns in the LG protractor neuron (shown schematically; STG). Note that the MCN1/CPN2 synaptic actions on the gastric mill CPG are omitted for clarity. The CoG and STG summaries are based on previous work (Coleman and Nusbaum, 1994; Norris et al., 1994; Beenhakker and Nusbaum, 2004; Stein et al., 2007; Blitz and Nusbaum, 2008, 2012; White and Nusbaum, 2011). The different LG burst patterns were retained by the LG-innervated muscles at the EJP and tension levels, despite these muscles having slow contraction and relaxation dynamics (Muscles), and by the lateral tooth protraction movements (Teeth) after triggering chewing patterns by mechanically stimulating the cardiac gutter (VCNs) or electrically stimulating the $\operatorname{coc}$ (POC neurons). EJPs, tension, and tooth movement traces are from actual recordings obtained for this paper. The image of the protracted teeth shows a screenshot from the endoscope camera. rLT, Right lateral tooth; ILT, left lateral tooth. Colored arrows indicate the flow of information from the extrinsic inputs through the STNS to the muscles and teeth. 
2012). These distinct patterns result largely from changes in the intrinsic and synaptic properties of the active circuit neurons, as well as the subset of neurons active during each motor pattern. Thus far, however, few studies have established whether different modulatory inputs also enable a defined network to drive distinct muscle and movement patterns in vivo (Zhurov et al., 2005; Proekt et al., 2007; Wu et al., 2010). In addition to providing further support for this relationship, the preservation of the distinct LG neuron burst patterns in vivo suggests that the previously established cellular and synaptic mechanisms underlying these distinct patterns in the isolated STNS (Beenhakker and Nusbaum, 2004; Blitz and Nusbaum, 2008, 2012) likely operate as well in the intact animal. Insofar as the most detailed mechanistic studies of circuit modulation are from invertebrate motor systems with slow muscle dynamics, confirmation that the resulting different motor patterns in the isolated nervous system not only persist in vivo but drive different movement patterns further establishes that circuit modulation is a pivotal behavior-generating event.

It was not a foregone conclusion that the distinct LG burst patterns in the isolated STNS would persist in vivo, given the numerous additional influences that have access to the gastric mill circuit in vivo from other CNS regions, sensory systems, and circulating hormones (Thuma et al., 2003; Kirby and Nusbaum, 2007; Marder and Bucher, 2007; Chen et al., 2009; Stein, 2009; Blitz and Nusbaum, 2011) as well as from the musculoskeletal system (Prochazka and Yakovenko, 2007; O'Connor et al., 2012). There were, as well, some notable changes in these gastric mill rhythms relative to their occurrence in the isolated STNS. For example, the POC-rhythm cycle period was considerably briefer in vivo (Blitz et al., 2008; White and Nusbaum, 2011; this paper). Additionally, the VCN retraction and protraction durations were approximately doubled and halved, respectively, in vivo relative to in vitro, yet there was no change in the cycle period. These and associated changes might have resulted from the presence of the aforementioned parallel influences present in vivo or from differences in the effectiveness of each stimulus under the two conditions. The latter possibility, however, seems unlikely to be significant. For example, the VCN-cycle period was unchanged under these two conditions, and the POC-triggered rhythms in the isolated STNS rarely if ever exhibit the relatively brief cycle periods that occurred in vivo, despite being elicited by a range of stimulus strengths from near threshold to supramaximal.

At least some of the rhythm changes in vivo likely resulted from the influence of sensory feedback, insofar as sensory feedback often mediates changes in burst duration and cycle period (Büschges, 2005; Pearson, 2008; Blitz and Nusbaum, 2011). For example, the prolonged VCN-retractor phase in vivo might have resulted from activity in the muscle stretch-sensitive gastropyloric receptor neurons, which are active during retraction and prolong this phase when stimulated in vitro (Katz et al., 1989; Birmingham et al., 1999; Beenhakker et al., 2005, 2007). Similarly, the anterior gastric receptor, a muscle sensory neuron, can shorten the LG neuron burst duration, as occurred in our in vivo recordings (Smarandache et al., 2008). The fact that, despite changes in several rhythm parameters in vivo, the distinct VCN and POC protraction patterns were preserved suggests that they play important and distinct behavioral roles. However, insofar as our in vivo experiments were performed in the explicit absence of food in the stomach, to enable clear video recordings of the tooth movements, determining the roles of smooth versus fast rhythmic protraction movements of the lateral teeth will require a different approach in future studies (McGaw, 2007).
Previous studies have established that, while muscles commonly generate contraction patterns that accurately mimic their neuronal input pattern, this is not always the case (Hooper and Weaver, 2000). This is particularly well established for muscles that exhibit slow contraction and relaxation dynamics relative to their input patterns (Morris et al., 2000; Thuma et al., 2003; Zhurov et al., 2005; Hooper et al., 2007). Contraction in these muscles can include sufficient summation across rhythmically repeating motor neuron bursts to minimize the ability of these muscles to generate discrete contractions in response to a relatively fast rhythmic input. Interestingly, other such muscles, even ones innervated by the same motor neuron, do have contraction and relaxation properties that enable them to effectively represent their fast rhythmic input (Hooper and Weaver, 2000; Thuma et al., 2003). Muscle responses to neuronal input can also be modified by the "catch" property of muscles (a nonlinear response to changing motor neuron input) and other active muscle properties. For example, muscles can produce myogenic contractions, or contract in response to stretch (Hooper and Weaver, 2000). However, muscles with slow dynamics do not necessarily filter fast rhythmic input patterns into tonic contractions (Morris and Hooper, 1997, 1998; Morris et al., 2000; Thuma et al., 2003; Zhurov et al., 2005; Zhurov and Brezina, 2006; Rosenbaum et al., 2010), and catch can also enable a strong representation of fast frequencies even in slow muscles (Hooper and Weaver, 2000).

The detailed muscle dynamics of the gastric mill system are unknown, but previous studies indicate that at least some gastric mill muscle-tension responses correspond to the relatively slow gastric mill cycle period (Stein et al., 2006). Before the present study, little information was available regarding how slowly contracting muscles respond to the distinct within-burst motor neuron patterns that can occur during different versions of a behavior. The distinct LG burst patterns during the VCN and POC rhythms are a particularly informative case study insofar as the pause between each LG burstlet is brief $(\sim 200 \mathrm{~ms})$, relative to the LG burst duration ( $~ 5.5 \mathrm{~s})$, during the POC rhythm (White and Nusbaum, 2011).

The finding that a LG-innervated muscle generates different tension patterns during these two gastric mill rhythms was not sufficient by itself for us to conclude that these different rhythms cause distinct movements during chewing. This limitation results partly from the fact that movements occurring in vivo are commonly a dynamic consequence of the degree of contraction in multiple agonist and antagonist muscles. Additionally, the muscles in this system move the teeth indirectly (Turrigiano and Heinzel, 1992). Interposed between the muscles and teeth are ossicles (cartilaginous support structures), and the quantitative relationship between muscle contraction and tooth movement remains to be determined (Hobbs and Hooper, 2009). Nonetheless, previous work combining video recording of teeth movements plus nerve recordings of LG activity supported the likelihood that distinct gm6ab tension patterns would contribute to different chewing movements (Heinzel et al., 1993). At the behavioral level, such distinct chewing patterns likely help optimize the digestion of the omnivorous diet of these animals, as they ingest numerous different hard-bodied and soft-bodied species (Stehlik, 1993; Donahue et al., 2009).

These results extend our appreciation of the role of neural circuit modulation from the isolated nervous system to the intact animal. Our previous work showed that selectively stimulating either of two identified pathways (VCNs, POC neurons) in vitro differently modulates the activity of the same identified projection neurons, which then drive different rhythmic motor pat- 
terns from the same identified CPG (Fig. 10; Beenhakker and Nusbaum, 2004; Blitz and Nusbaum, 2008, 2012; Blitz et al., 2008; White and Nusbaum, 2011). In this paper, we show that these different rhythmic motor patterns remain distinct in vivo (Fig. 10), indicating that the multifunctional nature of CPGs in the isolated nervous system can be a faithful representation of their nature in the intact animal. The fact that these distinct neural patterns drive different muscle and movement patterns, despite acting via muscles with slow contraction and relaxation dynamics, also suggests that the cellular and synaptic mechanisms underlying neural circuit modulation in the isolated nervous system can be pivotal to determining behavioral output, despite the presence of numerous additional influences in the intact animal.

\section{References}

Beenhakker MP, Nusbaum MP (2004) Mechanosensory activation of a motor circuit by coactivation of two projection neurons. J Neurosci 24:67416750. CrossRef Medline

Beenhakker MP, Blitz DM, Nusbaum MP (2004) Long-lasting activation of rhythmic neuronal activity by a novel mechanosensory system in the crustacean stomatogastric nervous system. J Neurophysiol 91:78-91. Medline

Beenhakker MP, DeLong ND, Saideman SR, Nadim F, Nusbaum MP (2005) Proprioceptor regulation of motor circuit activity by presynaptic inhibition of a modulatory projection neuron. J Neurosci 25:8794-8806. CrossRef Medline

Beenhakker MP, Kirby MS, Nusbaum MP (2007) Mechanosensory gating of proprioceptor input to modulatory projection neurons. J Neurosci 27:14308-14316. CrossRef Medline

Berkowitz A, Roberts A, Soffe SR (2010) Roles for multifunctional and specialized spinal interneurons during motor pattern generation in tadpoles, zebrafish larvae, and turtles. Front Behav Neurosci 4:36. CrossRef Medline

Birmingham JT, Szuts ZB, Abbott LF, Marder E (1999) Encoding of muscle movement on two time scales by a sensory neuron that switches between spiking and bursting modes. J Neurophysiol 82:2786-2797. Medline

Blitz DM, Nusbaum MP (2008) State-dependent presynaptic inhibition regulates central pattern generator feedback to descending inputs. J Neurosci 28:9564-9574. CrossRef Medline

Blitz DM, Nusbaum MP (2011) Neural circuit flexibility in a small sensorimotor system. Curr Opin Neurobiol 21:544-552. CrossRef Medline

Blitz DM, Nusbaum MP (2012) Modulation of circuit feedback specifies motor circuit output. J Neurosci 32:9182-9193. CrossRef Medline

Blitz DM, White RS, Saideman SR, Cook A, Christie AE, Nadim F, Nusbaum MP (2008) A newly identified extrinsic input triggers a distinct gastric mill rhythm via activation of modulatory projection neurons. J Exp Biol 211:1000-1011. CrossRef Medline

Bucher D, Taylor AL, Marder E (2006) Central pattern generating neurons simultaneously express fast and slow rhythmic activities in the stomatogastric ganglion. J Neurophysiol 95:3617-3632. CrossRef Medline

Büschges A (2005) Sensory control and organization of neural networks mediating coordination of multisegmental organs for locomotion. J Neurophysiol 93:1127-1135. Medline

Carrier DR (1989) Ventilatory action of the hypaxial muscles of the lizard Iguana iguana: a function of slow muscle. J Exp Biol 143:435-457. Medline

Chen R, Ma M, Hui L, Zhang J, Li L (2009) Measurement of neuropeptides in crustacean hemolymph via MALDI mass spectrometry. J Am Soc Mass Spectrum 20:708-718. CrossRef Medline

Coleman MJ, Nusbaum MP (1994) Functional consequences of compartmentalization of synaptic input. J Neurosci 14:6544-6552. Medline

Dickinson PS (2006) Neuromodulation of central pattern generators in invertebrates and vertebrates. Curr Opin Neurobiol 16:604-614. CrossRef Medline

Doi A, Ramirez JM (2008) Neuromodulation and the orchestration of the respiratory rhythm. Respir Physiol Neurobiol 164:96-104. CrossRef Medline

Donahue MJ, Nichols A, Santamaria CA, League-Pike PE, Krediet CJ, Perez KO, Shulman MJ (2009) Predation risk, prey abundance, and the verti- cal distribution of three brachyuran crabs on Gulf of Maine shores. J Crust Biol 29:523-531. CrossRef

Fahrmeir L (1992) Posterior mode estimation by extended Kalman filtering for multivariate dynamic generalized linear models. J Am Stat Assoc 87: 501-509. CrossRef

Harris-Warrick RM (2011) Neuromodulation and flexibility in central pattern generator networks. Curr Opin Neurobiol 21:685-692. CrossRef Medline

Hedrich UB, Stein W (2008) Characterization of a descending pathway: activation and effects on motor patterns in the brachyuran crustacean stomatogastric nervous system. J Exp Biol 211:2624-2637. CrossRef Medline

Hedrich UB, Diehl F, Stein W (2011) Gastric and pyloric motor pattern control by a modulatory projection neuron in the intact crab Cancer pagurus. J Neurophysiol 105:1671-1680. CrossRef Medline

Hedrick TL (2008) Software techniques for two- and three-dimensional kinematic measurements of biological and biomimetic systems. Bioinspir Biomim 3:034001. CrossRef Medline

Heinzel HG (1988a) Gastric mill activity in the lobster. I. Spontaneous modes of chewing. J Neurophysiol 59:528-550. Medline

Heinzel HG (1988b) Gastric mill activity in the lobster. II. Proctolin and octopamine initiate and modulate chewing. J Neurophysiol 59:551-565. Medline

Heinzel HG, Weimann JM, Marder E (1993) The behavioral repertoire of the gastric mill in the crab, Cancer pagurus: an in situ endoscopic and electrophysiological examination. J Neurosci 13:1793-1803. Medline

Hobbs KH, Hooper SL (2009) High-resolution computed tomography of lobster (Panulirus interruptus) stomach. J Morphol 270:1029-1041. CrossRef Medline

Hooper SL, Weaver AL (2000) Motor neuron activity is often insufficient to predict motor response. Curr Opin Neurobiol 10:676-682. CrossRef Medline

Hooper SL, O’Neil MB, Wagner R, Ewer J, Golowasch J, Marder E (1986) The innervation of the pyloric region of the crab, Cancer borealis: homologous muscles in decapod species are differently innervated. J Comp Physiol 159:227-240. CrossRef

Hooper SL, Guschlbauer C, von Uckermann G, Büschges A (2006) Natural neural output that produces highly variable locomotory movements. J Neurophysiol 96:2072-2088. CrossRef Medline

Hooper SL, Guschlbauer C, von Uckermann G, Büschges A (2007) Different motor neuron spike patterns produce contractions with very similar rises in graded slow muscles. J Neurophysiol 97:1428-1444. Medline

Katz PS, Eigg MH, Harris-Warrick RM (1989) Serotonergic/cholinergic muscle receptor cells in the crab stomatogastric nervous system. I. Identification and characterization of the gastropyloric receptor cells. J Neurophysiol 62:558-570. Medline

Kiehn O, Kjaerulff O (1996) Spatiotemporal characteristics of 5-HT and dopamine-induced rhythmic hindlimb activity in the in vitro neonatal rat. J Neurophysiol 75:1472-1482. Medline

Kilman VL, Marder E (1996) Ultrastructure of the stomatogastric ganglion neuropil of the crab, Cancer borealis. J Comp Neurol 374:362-375. CrossRef Medline

Kirby MS, Nusbaum MP (2007) Peptide hormone modulation of a neuronally modulated motor circuit. J Neurophysiol 98:3206-3220. CrossRef Medline

Marder E (2012) Neuromodulation of neuronal circuits: back to the future. Neuron 76:1-11. CrossRef Medline

Marder E, Bucher D (2001) Central pattern generators and the control of rhythmic movements. Curr Biol 11:R986-R996. CrossRef Medline

Marder E, Bucher D (2007) Understanding circuit dynamics using the stomatogastric nervous system of lobsters and crabs. Annu Rev Physiol 69: 291-316. CrossRef Medline

Marder E, Calabrese RL (1996) Principles of rhythmic motor pattern generation. Physiol Rev 76:687-717. Medline

Marder E, Bucher D, Schulz DJ, Taylor AL (2005) Invertebrate central pattern generation moves along. Curr Biol 15:R685-R699. CrossRef Medline

Maynard DM, Dando MR (1974) The structure of the stomatogastric neuromuscular system in Callinectes sapidus, Homarus americanus and Panulirus argus (Decapoda Crustacea). Philos Trans R Soc Lond B Biol Sci 268:161-220. CrossRef Medline

McGaw IJ (2007) The interactive effects of exercise and feeding on oxygen uptake, activity levels, and gastric processing in the graceful crab Cancer gracilis. Physiol Biochem Zool 80:335-343. CrossRef Medline 
Morris LG, Hooper SL (1997) Muscle response to changing neuronal input in the lobster (Panulirus interruptus) stomatogastric system: spike number- versus spike frequency-dependent domains. J Neurosci 17: 5956-5971. Medline

Morris LG, Hooper SL (1998) Muscle response to changing neuronal input in the lobster (Panulirus interruptus) stomatogastric system: slow muscle properties can transform rhythmic input into tonic output. J Neurosci 18:3433-3442. Medline

Morris LG, Thuma JB, Hooper SL (2000) Muscles express motor pattern of noninnervating neural networks by filtering broad-band input. Nat Neurosci 3:245-250. CrossRef Medline

Norris BJ, Coleman MJ, Nusbaum MP (1994) Recruitment of a projection neuron determines gastric mill motor pattern selection in the stomatogastric nervous system of the crab, Cancer borealis. J Neurophysiol 72: 1451-1463. Medline

Nusbaum MP, Blitz DM (2012) Neuropeptide modulation of microcircuits. Curr Opin Neurobiol 22:592-601. CrossRef Medline

O'Connor R, Segers LS, Morris KF, Nuding SC, Pitts T, Bolser DC, Davenport PW, Lindsey BG (2012) A joint computational respiratory neural network-biomechanical model for breathing and airway defensive behaviors. Front Physiol 3:264. CrossRef Medline

Pearson J (1908) Cancer crabs. In: Liverpool Marine Biology Committee Memoirs: on typical British marine plants and animals (Herdman WA, ed). London: Williams and Norgate.

Pearson KG (2008) Role of sensory feedback in the control of stance duration in walking cats. Brain Res Rev 57:222-227. CrossRef Medline

Pillow JW, Ahmadian Y, Paninski L (2011) Model-based decoding, information estimation, and change-point detection techniques for multineuron spike-trains. Neur Comp 23:1-45. CrossRef Medline

Prochazka A, Yakovenko S (2007) The neuromechanical tuning hypothesis. Prog Brain Res 165:255-265. CrossRef Medline

Proekt A, Jing J, Weiss KR (2007) Multiple contributions of an inputrepresenting neuron to the dynamics of the Aplysia feeding network. J Neurophysiol 97:3046-3056. CrossRef Medline

Rosenbaum P, Wosnitza A, Büschges A, Gruhn M (2010) Activity patterns and timing of muscle activity in the forward walking and backward walking stick insect Carausius morosus. J Neurophysiol 104:1681-1695. CrossRef Medline

Smarandache CR, Daur N, Hedrich UB, Stein W (2008) Regulation of motor pattern frequency by reversals in proprioceptive feedback. Eur J Neurosci 28:460-474. CrossRef Medline

Stehlik LL (1993) Diets of the brachyuran crabs Cancer irroratus, C. borealis, and Ovalipes ocellatus in the New York Bight. J Crust Biol 13:723-735. CrossRef
Stein W (2009) Modulation of stomatogastric rhythms. J Comp Physiol A Neuroethol Sens Neural Behav Physiol 195:989-1009. CrossRef Medline

Stein W, Smarandache CR, Nickmann M, Hedrich UB (2006) Functional consequences of activity-dependent synaptic enhancement at a crustacean neuromuscular junction. J Exp Biol 209:1285-1300. CrossRef Medline

Stein W, DeLong ND, Wood DE, Nusbaum MP (2007) Divergent cotransmitter actions underlie motor pattern activation by a modulatory projection neuron. Eur J Neurosci 26:1148-1165. CrossRef Medline

Thuma JB, Morris LG, Weaver AL, Hooper SL (2003) Lobster (Panulirus interruptus) pyloric muscles express the motor patterns of three neural networks, only one of which innervates the muscles. J Neurosci 23:89118920. Medline

Turrigiano GG, Heinzel H-G (1992) Behavioral correlates of stomatogastric network function. In: Dynamic biological networks, the stomatogastric nervous system (Harris-Warrick RM, Marder E, Selverston AI, Moulins M, eds), pp 197-220. Cambridge, MA: MIT.

Weimann JM, Meyrand P, Marder E (1991) Neurons that form multiple pattern generators: identification and multiple activity patterns of gastric/ pyloric neurons in the crab stomatogastric system. J Neurophysiol 65: 111-122. Medline

Wenning A, Cymbalyuk GS, Calabrese RL (2004) Heartbeat control in leeches. I. Constriction pattern and neural modulation of blood pressure in intact animals. J Neurophysiol 91:382-396. Medline

White RS, Nusbaum MP (2011) The same core rhythm generator underlies different rhythmic motor patterns. J Neurosci 31:11484-11494. CrossRef Medline

Wood DE, Stein W, Nusbaum MP (2000) Projection neurons with shared cotransmitters elicit different motor patterns from the same neural circuit. J Neurosci 20:8943-8953. Medline

Wood DE, Manor Y, Nadim F, Nusbaum MP (2004) Intercircuit control via rhythmic regulation of projection neuron activity. J Neurosci 24:74557463. CrossRef Medline

Wu JS, Vilim FS, Hatcher NG, Due MR, Sweedler JV, Weiss KR, Jing J (2010) Composite modulatory feedforward loop contributes to the establishment of a network state. J Neurophysiol 103:2174-2184. CrossRef Medline

Zhurov Y, Brezina V (2006) Variability of motor neuron spike timing maintains and shapes contractions of the accessory radula closer muscle of Aplysia. J Neurosci 26:7056-7070. CrossRef Medline

Zhurov Y, Proekt A, Weiss KR, Brezina V (2005) Changes of internal state are expressed in coherent shifts of neuromuscular activity in Aplysia feeding behavior. J Neurosci 25:1268-1280. CrossRef Medline 